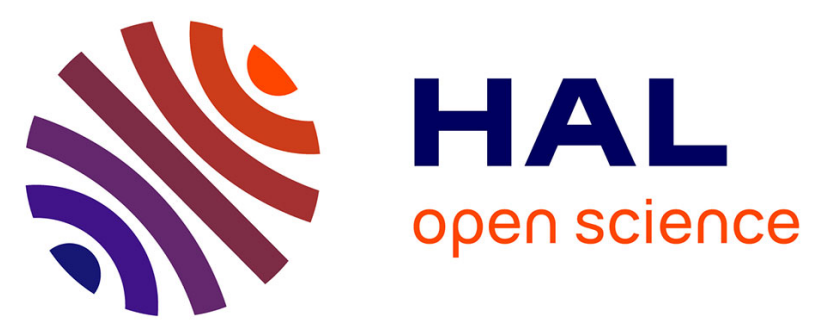

\title{
An entropy satisfying two-speed relaxation system for the barotropic Euler equations. Application to the numerical approximation of low Mach number flows.
}

François Bouchut, Christophe Chalons, Sébastien Guisset

\section{- To cite this version:}

François Bouchut, Christophe Chalons, Sébastien Guisset. An entropy satisfying two-speed relaxation system for the barotropic Euler equations. Application to the numerical approximation of low Mach number flows.. Numerische Mathematik, 2020, 145, pp.35-76. 10.1007/s00211-020-01111-5 . hal$01661275 \mathrm{v} 2$

\section{HAL Id: hal-01661275 \\ https://hal.science/hal-01661275v2}

Submitted on 23 Mar 2020

HAL is a multi-disciplinary open access archive for the deposit and dissemination of scientific research documents, whether they are published or not. The documents may come from teaching and research institutions in France or abroad, or from public or private research centers.
L'archive ouverte pluridisciplinaire HAL, est destinée au dépôt et à la diffusion de documents scientifiques de niveau recherche, publiés ou non, émanant des établissements d'enseignement et de recherche français ou étrangers, des laboratoires publics ou privés. 


\title{
An entropy satisfying two-speed relaxation system for the barotropic Euler equations. Application to the numerical approximation of low Mach number flows
}

\author{
François Bouchut ${ }^{1}$, Christophe Chalons ${ }^{2}$, Sébastien Guisset ${ }^{2}$ \\ ${ }^{1}$ Université Paris-Est, Laboratoire d'Analyse et de Mathématiques \\ Appliquées (UMR 8050), \\ CNRS, UPEM, UPEC, F-77454, Marne-la-Vallée, France \\ ${ }^{2}$ Laboratoire de Mathématiques de Versailles, UVSQ, CNRS, Université \\ Paris-Saclay, F-78035 Versailles, France
}

\begin{abstract}
In the first part of this work, we introduce a new relaxation system in order to approximate the solutions to the barotropic Euler equations. We show that the solutions to this two-speed relaxation model can be understood as viscous approximations of the solutions to the barotropic Euler equations under appropriate sub-characteristic conditions. Our relaxation system is a generalization of the well-known Suliciu relaxation system, and it is entropy satisfying. A Godunovtype finite volume scheme based on the exact resolution of the Riemann problem associated with the relaxation system is deduced, as well as its stability properties. In the second part of this work, we show how the new relaxation approach can be successfully applied to the numerical approximation of low Mach number flows. We prove that the underlying scheme satisfies the well-known asymptotic-preserving property in the sense that it is uniformly (first-order) accurate with respect to the Mach number, and at the same time it satisfies a fully discrete entropy inequality. This discrete entropy inequality allows us to prove strong stability properties in the low Mach regime. At last, numerical experiments are given to illustrate the behaviour of our scheme.
\end{abstract}

KEYwords. Barotropic Euler equations, low Mach number regime, relaxation system, entropy inequality.

\section{Introduction}

Relaxation approximations to hyperbolic systems of conservation laws have been introduced in [33, 16, 42], see also [36, 37]. They allow to design cheap 
and accurate Godunov-type finite volume methods. Moreover they can be designed to satisfy an entropy inequality. These methods are based on the knowledge of the Riemann solution to the relaxation system, and can be also interpreted in the well-known Harten Lax and Van Leer's framework of approximate Riemann solvers [31, 4, 3. Without any attempt to be exhaustive, we also refer the reader to [18, 6, 1, 13, 10, 11] and the references therein. A class of relaxation approximations to fluid systems is derived from Suliciu's one [42]. In these relaxation systems the main idea, related to the particular fluid structure [26], basically consists in linearizing the flux function, and more precisely the underlying pressure term, which encompasses the genuine nonlinearities of the model, while keeping unchanged the the convective part of the system and the linearly degenerate characteristic field. This procedure enables the derivation of an extended relaxation system which is consistent with both the original system and its entropy inequality in the limit of a vanishing relaxation parameter. By extended, we mean that it contains additional variables associated with the linearization process. The relaxation models are hyperbolic with only linearly degenerated characteristic fields, so that the associated Riemann problem is easy to solve. This makes the whole procedure particularly adapted to construct efficient Godunov-type methods based on approximate Riemann solvers, see $[18,4$, 2, 41, 8, 19, 5, 9].

In the same spirit, we propose here a new relaxation approximation of the barotropic Euler equations based on the relaxation of both the pressure and velocity variables, leading to an enlarged relaxation system made of two additional relaxation variables, instead of one usually in the Suliciu spirit. We thus have two velocities, one for the conserved momentum and one for the convective fluxes. This idea to have two velocities was indeed present in [28. The two relaxation variables (pressure and velocity) are associated with two relaxation speeds, which explains the two-speed relaxation system terminology. As usual they must satisfy subcharacteristic conditions for the scheme to be entropy satisfying. With these two speeds we are able to monitor independently the numerical viscosity on the density and velocity variables. This non-standard relaxation approach provides us with a new entropy satisfying Riemann solver that generalizes the classical one, and which could be well-adapted to several situations because of its ability to design a particular shape for the numerical diffusion matrix. In the present work, we are especially interested in the situation of low Mach numbers.

Let us briefly recall that two important issues naturally arise when considering low Mach number flows. The first one is concerned with the now very well-known excessive numerical diffusion of standard Godunov-type schemes in the low Mach number regime, leading to poor numerical solutions for coarse meshes. By coarse meshes, we mean here that the size of the mesh is large compared to the mean Mach number of the flow. This topic has been the subject of many research works, see for instance 
44, 29, 17, 35, 30, 39, 21, 22, 20, 24, 23, and more recently [14, 15, 25, 43, 32, which most of the time address the problem by modifying the viscosity matrices of the schemes in order to reduce the numerical diffusion in low Mach number regimes. By doing so they give up with the entropy inequality.

The second issue is concerned with the CFL condition driving the time steps of the usual explicit in time Godunov-type solvers in the low Mach regime, i.e. when the fluid is slow compared to the acoustic waves. In such situation, the (fast) acoustic waves do not play an important role in the solution itself, but are responsible for very restrictive CFL conditions and therefore the use of very small timesteps for the sake of stability. Several implicit or implicit-explicit schemes which do not suffer from such restrictive CFL conditions have been proposed in the literature, see for instance [35, 21, 20, 22, 14, 15, 30, 27, 34 and the above references.

In the present work, it is shown that the proposed two-speed relaxation scheme enables to derive a new solver which is accurate in all Mach number regimes, while it keeps satisfied a fully discrete entropy inequality. Moreover the explicit CFL condition can be monitored to reduce to a parabolic-like CFL condition in the low Mach regime.

The outline of the paper is as follows. In Section 2, the two-speed relaxation model is introduced in order to approximate the solutions to the barotropic Euler equations. In particular, it is shown that the solutions of the relaxation model can be seen as a viscous approximation of the barotropic Euler system under appropriate subcharacteristic conditions on the underlying wave speeds. The same subcharacteristic conditions imply the existence of an extended entropy, ensuring the entropy inequality. A Godunov-type scheme based on the exact resolution of the Riemann problem to the relaxation model is then proposed. We prove that the scheme is positive and satisfies a discrete entropy inequality. In Section 3 we consider the low Mach number regime, and we prove that the two-speed relaxation scheme is asymptotic-preserving (i.e. it is uniformly first-order accurate with respect to the Mach number) for an adequate choice of the speeds. Moreover we prove that for small Mach number $M$ our scheme becomes consistent with the incompressible Euler equations, with particularly strong stability properties. Numerical results in $2 \mathrm{~d}$ are presented that confirm the theoretical properties. A conclusion is given in Section 4.

\section{A two-speed relaxation scheme for the barotropic Euler equations}

In this section, we first recall the governing equations of the barotropic Euler system. We then introduce our two-speed relaxation model in order to approximate the solutions. A Godunov-type finite volume scheme is then proposed, based on the exact resolution of the Riemann problem associated 
to the relaxation model. The stability properties of the relaxation scheme are established and a fully discrete entropy property is proved. Finally, a numerical experiment is presented to illustrate the effect of the choice of the speeds.

\subsection{The barotropic Euler equations}

We are interested in the very classical barotropic Euler equations given in one space dimension by

$$
\partial_{t}\left(\begin{array}{c}
\rho \\
\rho u
\end{array}\right)+\partial_{x}\left(\begin{array}{c}
\rho u \\
\rho u^{2}+p
\end{array}\right)=0, \quad x \in \mathbb{R}, \quad t>0,
$$

where the pressure law $p=p(\rho)$ is assumed to satisfy the monotonicity and convexity properties $p^{\prime}(\rho)>0$ and

$$
\frac{d}{d \rho}\left(\rho \sqrt{p^{\prime}(\rho)}\right)>0 \quad \text { for all } \rho>0 .
$$

Under these assumptions, this system is well-known to be hyperbolic with eigenvalues given by $\lambda_{ \pm}=u \pm c$, where the sound speed $c$ is given by $c=\sqrt{p^{\prime}(\rho)}$, with genuinely nonlinear characteristic fields. We will also assume that

$$
\frac{d}{d \rho}\left(\rho \sqrt{p^{\prime}(\rho)}\right) \leq \beta \sqrt{p^{\prime}(\rho)} \quad \text { for some constant } \beta>1 .
$$

Setting $U=(\rho, \rho u)^{T}$ and $F(U)=\left(\rho u, \rho u^{2}+p\right)^{T}$, the entropy inequality associated with (2.1) is given by

$$
\partial_{t} \eta(U)+\partial_{x} G(U) \leq 0,
$$

where the strictly convex entropy $U \rightarrow \eta(U)$ is nothing but the total energy defined by

$$
\eta(U)=\rho \frac{u^{2}}{2}+\rho e(\rho)
$$

with the internal energy $e(\rho)$ such that

$$
e^{\prime}(\rho)=\frac{p(\rho)}{\rho^{2}},
$$

and the associated entropy flux $U \rightarrow G(U)$ given by

$$
G(U)=(\eta(U)+p(\rho)) u .
$$

In particular, integrating (2.4) with vanishing conditions at $\pm \infty$ gives

$$
\frac{d}{d t} \int_{\mathbb{R}} \eta(U) d x \leq 0 .
$$




\subsection{A two-speed relaxation system}

We consider the approximation of the Euler system (2.1) by the following relaxation system,

$$
\begin{gathered}
\partial_{t} \rho+\partial_{x}(\rho v)=0 \\
\partial_{t}(\rho u)+\partial_{x}(\rho u v+\pi)=0, \\
\partial_{t}(\rho v)+\partial_{x}\left(\rho v^{2}\right)+\frac{a}{b} \partial_{x} \pi=\rho \frac{u-v}{\varepsilon}, \\
\partial_{t}(\rho \pi)+\partial_{x}(\rho \pi v)+a b \partial_{x} v=\rho \frac{p-\pi}{\varepsilon}, \\
\partial_{t} a+v \partial_{x} a=0, \\
\partial_{t} b+v \partial_{x} b=0,
\end{gathered}
$$

where $\pi$ and $v$ are two relaxation variables associated with the pressure $p=p(\rho)$ and the velocity $u$, and $a, b$ are positive quantities. When $a \equiv b$ and $v \equiv u$ we recover the usual Suliciu relaxation system. The positive constant $\varepsilon$ is the so-called relaxation parameter. We notice that as is usual, the equations (2.10), 2.11), 2.12 can be written in conservative form by using (2.8), as

$$
\begin{aligned}
\partial_{t} \frac{\rho v b}{a}+\partial_{x}\left(\rho v^{2} \frac{b}{a}+\pi\right) & =\frac{b}{a} \rho \frac{u-v}{\varepsilon} \\
\partial_{t}\left(\frac{\rho \pi}{a b}\right)+\partial_{x}\left(\rho \pi \frac{v}{a b}+v\right) & =\frac{1}{a b} \rho \frac{p-\pi}{\varepsilon} \\
\partial_{t}(\rho a)+\partial_{x}(\rho a v) & =0 \\
\partial_{t}(\rho b)+\partial_{x}(\rho b v) & =0 .
\end{aligned}
$$

We can remark that at least formally, in the asymptotic limit $\varepsilon \rightarrow 0$ we have $\pi=p$ and $v=u$ and we recover the original system (2.1). However and in order to prevent this relaxation process from instabilities, it is now well established that some subcharacteristic conditions must be imposed. Here, we will see that these conditions are concerned with $a$ and $b$ and express that they must be sufficiently large. This issue will be made more precise in the next subsection.

From the system (2.8)-2.12 one can easily derive the following equations,

$$
\begin{gathered}
\rho\left(\partial_{t} u+v \partial_{x} u\right)+\partial_{x} \pi=0, \\
\rho\left(\partial_{t} v+v \partial_{x} v\right)+\frac{a}{b} \partial_{x} \pi=\rho \frac{u-v}{\varepsilon}, \\
\rho\left(\partial_{t} \pi+v \partial_{x} \pi\right)+a b \partial_{x} v=\rho \frac{p-\pi}{\varepsilon} .
\end{gathered}
$$

We deduce the quasi diagonal form of the system

$$
\partial_{t}(\pi+b v)+(v+a / \rho) \partial_{x}(\pi+b v)-\frac{a v}{\rho} \partial_{x} b=\frac{p-\pi+b(u-v)}{\varepsilon},
$$




$$
\begin{aligned}
\partial_{t}(\pi-b v)+(v-a / \rho) \partial_{x}(\pi-b v)-\frac{a v}{\rho} \partial_{x} b & =\frac{p-\pi-b(u-v)}{\varepsilon}, \\
\partial_{t}\left(\frac{1}{\rho}+\frac{\pi}{a b}\right)+v \partial_{x}\left(\frac{1}{\rho}+\frac{\pi}{a b}\right) & =\frac{1}{a b} \frac{p-\pi}{\varepsilon} \\
\partial_{t}\left(u-\frac{b}{a} v\right)+v \partial_{x}\left(u-\frac{b}{a} v\right) & =-\frac{b}{a} \frac{u-v}{\varepsilon}
\end{aligned}
$$

completed with $(2.12)$. Therefore, it can be easily proved that the relaxation system (2.8)-2.12) is hyperbolic with three eigenvalues given by

$$
\lambda_{ \pm}^{r}=v \pm a / \rho, \quad \lambda_{0}=v,
$$

the latter having multiplicity 4 . Note that $\lambda_{ \pm}^{r}$ are nothing but approximations of the exact eigenvalues $\lambda_{ \pm}$. More importantly, the characteristic fields associated with these eigenvalues are all linearly degenerate, which allows to solve analytically the Riemann problem associated with the left-hand side of (2.8)-2.12). This will be the purpose of subsection 2.4. Note that this property is sufficient to justify the introduction of our relaxation system to approximate the solutions to the barotropic Euler equations.

\section{$2.3 \quad$ Viscous approximation}

In this paragraph, we prove that the solutions of the two-speed relaxation system (2.8)-2.12 can be seen as a viscous approximation of the solution of the barotropic Euler system (2.1) under suitable conditions on the wave speeds $a$ and $b$. We start noticing that from (2.10), (2.11) it follows

$$
\left\{\begin{array}{l}
v=u-\frac{\varepsilon}{\rho}\left(\partial_{t}(\rho v)+\partial_{x}\left(\rho v^{2}\right)+\frac{a}{b} \partial_{x} \pi\right), \\
\pi=p-\frac{\varepsilon}{\rho}\left(\partial_{t}(\rho \pi)+\partial_{x}(\rho \pi v)+a b \partial_{x} v\right) .
\end{array}\right.
$$

By inserting (2.24) into (2.8) and (2.9) one obtains

$$
\begin{aligned}
\partial_{t} \rho+ & \partial_{x}(\rho u)-\varepsilon \partial_{x}\left(\partial_{t}(\rho u)+\partial_{x}\left(\rho u^{2}\right)+\frac{a}{b} \partial_{x} p\right)=\mathcal{O}\left(\varepsilon^{2}\right), \\
\partial_{t}(\rho u) & +\partial_{x}\left(\rho u^{2}+p\right) \\
& -\varepsilon \partial_{x}\left(u \partial_{t}(\rho u)+u \partial_{x}\left(\rho u^{2}\right)+u \frac{a}{b} \partial_{x} p\right) \\
& -\varepsilon \partial_{x}\left(\frac{1}{\rho} \partial_{t}(\rho p)+\frac{1}{\rho} \partial_{x}(\rho p u)+\frac{a b}{\rho} \partial_{x} u\right)=\mathcal{O}\left(\varepsilon^{2}\right) .
\end{aligned}
$$

Using now the fact that

$$
\begin{aligned}
& \partial_{t}(\rho u)=-\partial_{x}\left(\rho u^{2}\right)-\partial_{x} p+\mathcal{O}(\varepsilon), \\
& \partial_{t}(\rho p)=-\left(p(\rho)+\rho p^{\prime}(\rho)\right) \partial_{x}(\rho u)+\mathcal{O}(\varepsilon)=-\partial_{x}(\rho p u)-\rho^{2} p^{\prime}(\rho) \partial_{x} u+\mathcal{O}(\varepsilon),
\end{aligned}
$$


one obtains the system

$$
\partial_{t}\left(\begin{array}{c}
\rho \\
\rho u
\end{array}\right)+\partial_{x}\left(\begin{array}{c}
\rho u \\
\rho u^{2}+p
\end{array}\right)-\varepsilon \partial_{x}\left(D \partial_{x}\left(\begin{array}{c}
\rho \\
\rho u
\end{array}\right)\right)=\mathcal{O}\left(\varepsilon^{2}\right),
$$

with

$$
D=\left(\begin{array}{cc}
\left(\frac{a}{b}-1\right) p^{\prime}(\rho) & 0 \\
u\left(\frac{a}{b}-1\right) p^{\prime}(\rho)-u\left(\frac{a b}{\rho^{2}}-p^{\prime}(\rho)\right) & \frac{a b}{\rho^{2}}-p^{\prime}(\rho)
\end{array}\right) .
$$

Therefore the solutions of the two-speed relaxation model (2.8)-2.12 can be seen as a viscous approximation of the solutions of the barotropic Euler system (2.1) as soon as the viscosity matrix (2.28) has nonnegative eigenvalues, which holds true under the subcharacteristic conditions

$$
a \geq b, \quad a b \geq \rho^{2} p^{\prime}(\rho)=\rho^{2} c^{2} .
$$

One can check moreover that $D^{T} \eta^{\prime \prime}(U)$ is symmetric with $\eta$ the entropy given by (2.5), a condition of entropy compatibility of the viscous system (2.27), see [3]. It is interesting to note that in the case $a=b$, which corresponds to the usual Suliciu relaxation approximation of the barotropic Euler equations, see for instance [4], one gets the usual subcharacteristic condition $a^{2} \geq \rho^{2} c^{2}$. It is thus clear from (2.28) that the new relaxation procedure adds numerical diffusion on the density variable. This property will be useful to understand the low Mach number regime in Section 3. It is also interesting to notice that the relaxation system (2.8)-2.12) appears exactly under the form of an entropy compatible viscous system, those being characterized in [28].

\subsection{Riemann problem}

We give in this section the solution to the Riemann problem associated with the left-hand side of the relaxation system $(2.8)-(2.12)$. Let us denote $U^{r}$ the unknown vector ("r" stands here for "relaxation") and recall that the Riemann problem consists in solving the governing equations when considering an initial data made of two constant states $U^{r, L}$ on the left and $U^{r, R}$ on the right, separated by an initial discontinuity located at $x=0$. Since the system under consideration is hyperbolic with three linearly degenerate characteristic fields, the exact Riemann solutions are self-similar and made of three contact discontinuities propagating with velocities $\sigma^{L}=\lambda_{-}^{r}\left(U^{r, L}\right)=\lambda_{-}^{r}\left(U^{r, L *}\right)$, $\sigma^{*}=\lambda_{0}^{r}\left(U^{r, L *}\right)=\lambda_{0}^{r}\left(U^{r, R *}\right), \sigma^{R}=\lambda_{+}^{r}\left(U^{r, R *}\right)=\lambda_{+}^{r}\left(U^{r, R}\right)$ and separating two intermediates states $U^{r, L *}$ on the left and $U^{r, R *}$ on the right. We will assume here that

$$
\sigma^{L}<\sigma^{*}<\sigma^{R}
$$


which will be proved in subsection 2.6.3 under appropriate choices of $a$ and $b$. We thus have

$$
U^{r}\left(x / t ; U^{r, L}, U^{r, R}\right)=\left\{\begin{array}{rll}
U^{r, L} & \text { if } & \frac{x}{t}<\sigma^{L}, \\
U^{r, L *} & \text { if } & \sigma^{L}<\frac{x}{t}<\sigma^{*}, \\
U^{r, R *} & \text { if } & \sigma^{*}<\frac{x}{t}<\sigma^{R}, \\
U^{r, R} & \text { if } & \sigma^{R}<\frac{x}{t} .
\end{array}\right.
$$

This usual structure is displayed on Figure 1. Denoting by upper indices $L *, R *$ the associated values, the intermediate states $U^{r, L *}$ and $U^{r, R *}$ are obtained from the following relations,

$$
\begin{aligned}
& \pi^{L \star}=\pi^{R \star} \equiv \pi^{\star}=\frac{b^{R} \pi^{L}+b^{L} \pi^{R}+b^{L} b^{R}\left(v^{L}-v^{R}\right)}{b^{L}+b^{R}}, \\
& v^{L \star}=v^{R \star} \equiv v^{\star}=\frac{b^{L} v^{L}+b^{R} v^{R}+\pi^{L}-\pi^{R}}{b^{L}+b^{R}}, \\
& \frac{1}{\rho^{L \star}}=\frac{1}{\rho^{L}}+\frac{b^{R}\left(v^{R}-v^{L}\right)+\pi^{L}-\pi^{R}}{a^{L}\left(b^{L}+b^{R}\right)}, \\
& \frac{1}{\rho^{R \star}}=\frac{1}{\rho^{R}}+\frac{b^{L}\left(v^{R}-v^{L}\right)+\pi^{R}-\pi^{L}}{a^{R}\left(b^{L}+b^{R}\right)}, \\
& u^{L \star}=u^{L}+\frac{b^{L}}{a^{L}\left(b^{L}+b^{R}\right)}\left(b^{R}\left(v^{R}-v^{L}\right)+\pi^{L}-\pi^{R}\right), \\
& u^{R \star}=u^{R}+\frac{b^{R}}{a^{R}\left(b^{L}+b^{R}\right)}\left(b^{L}\left(v^{L}-v^{R}\right)+\pi^{L}-\pi^{R}\right), \\
& a^{L \star}=a^{L}, \quad a^{R \star}=a^{R}, b^{L \star}=b^{L}, \quad b^{R \star}=b^{R} .
\end{aligned}
$$

These formulas are obtained by writing that the Riemann invariants associated with each characteristic field are constant across the associated discontinuity. The Rieman invariants for the $\lambda_{0}^{r}$ wave are $v, \pi$ (setting $\partial_{t}+v \partial_{x}=0$ in (2.8)-(2.12), it remains only $\partial_{x} \pi=0$ and $\left.\partial_{x} v=0\right)$. According to (2.12) and (2.19)-(2.22), the Riemann invariants for the $\lambda_{ \pm}^{r}$ wave are $a, b, \pi \mp b v$, $1 / \rho+\pi /(a b), u-(b / a) v$. These formulas are completed by the values of the speeds

$$
\sigma^{L}=v^{L}-\frac{a^{L}}{\rho^{L}}, \quad \sigma^{*}=v^{\star}, \quad \sigma^{R}=v^{R}+\frac{a^{R}}{\rho^{R}} .
$$

Writing $\sigma^{*}-\sigma^{L}=\lambda_{0}^{r}\left(U^{r, L *}\right)-\lambda_{-}^{r}\left(U^{r, L *}\right)=a^{L} / \rho^{L \star}$ and $\sigma^{R}-\sigma^{*}=\lambda_{+}^{r}\left(U^{r, R *}\right)-$ $\lambda_{0}^{r}\left(U^{r, R *}\right)=a^{R} / \rho^{R \star}$, we get that the right ordering (2.30) is equivalent to the positivity of the intermediate densities $\rho^{L \star}>0, \rho^{R \star}>0$.

\subsection{The relaxation scheme}

Hereafter and using very classical notations, space and time will be discretized using constant spacestep $\Delta x$ and timestep $\Delta t$. We will consider a set of cells $\kappa_{i}=\left(x_{i-1 / 2}, x_{i+1 / 2}\right)$ and instants $t^{n}=n \Delta t$, with $x_{i+1 / 2}-x_{i-1 / 2}=$ 


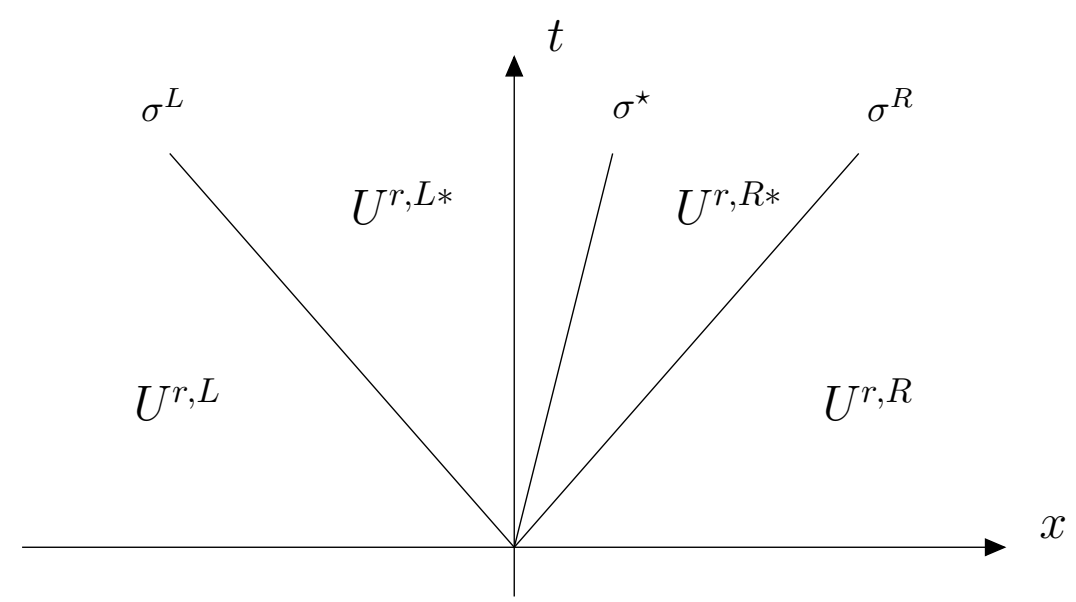

Figure 1: Structure of the solution to the relaxation Riemann problem

$\Delta x$ and $x_{i}=\left(x_{i-1 / 2}+x_{i+1 / 2}\right) / 2$ for $i \in \mathbb{Z}$ and $n \in \mathbb{N}$.

Considering the finite volume framework, we aim at computing a piecewise constant approximation on each cell of the exact solution $U\left(t^{n}, x\right)$ to (2.1) and we denote it $U_{i}^{n}$ for all $i \in \mathbb{Z}$ and $n \in \mathbb{N}$, so that

$$
U_{i}^{n} \approx \frac{1}{\Delta x} \int_{x_{i-1 / 2}}^{x_{i+1 / 2}} U\left(t^{n}, x\right) d x
$$

Assuming $\left(U_{i}^{n}\right)_{i \in \mathbb{Z}}$ we update it to the next time level $t^{n+1}$ using the above two-speed relaxation system. The procedure is very classical and amounts to first define piecewise constant approximations $U_{i}^{r, n}$ of the relaxation system that we take at equilibrium, that is to say such that

$$
\pi_{i}^{n}=p\left(\rho_{i}^{n}\right), \quad v_{i}^{n}=u_{i}^{n} \quad \text { for all } i .
$$

A choice of $a^{L}, a^{R}, b^{L}, b^{R}$ has to be done also, in order to satisfy the positivity of density and an entropy inequality. this will be discussed in Subsection 2.6. Then we use the standard Godunov-relaxation approach [4] which means that we solve 2.8)-2.12 without right-hand side for $t \in$ $\left(t^{n}, t^{n+1}\right)$, and we finally retain only the components $\rho, \rho u$ in the solution, that we average over a cell to get $U_{i}^{n+1}$. Using the notations of the previous section, we end up with the following conservative numerical scheme

$$
\begin{gathered}
U_{i}^{n+1}=U_{i}^{n}-\frac{\Delta t}{\Delta x}\left(F_{i+1 / 2}^{n}-F_{i-1 / 2}^{n}\right), \\
F_{i+1 / 2}^{n}=F\left(U_{i}^{n}, U_{i+1}^{n}\right),
\end{gathered}
$$


where the numerical flux is given by

$$
F\left(U^{L}, U^{R}\right)= \begin{cases}F\left(U^{L}\right) & \text { if } \sigma^{L} \geq 0 \\ F^{L \star} & \text { if } \sigma^{L} \leq 0 \leq \sigma^{*} \\ F^{R \star} & \text { if } \sigma^{*} \leq 0 \leq \sigma^{R} \\ F\left(U^{R}\right) & \text { if } \sigma^{R} \leq 0\end{cases}
$$

Because of the conservative equations (2.8), 2.9), the intermediate fluxes can be written as

$$
\begin{aligned}
& F^{L \star}=\left(\rho^{L \star} v^{\star}, \rho^{L \star} u^{L \star} v^{\star}+\pi^{\star}\right), \\
& F^{R \star}=\left(\rho^{R \star} v^{\star}, \rho^{R \star} u^{R \star} v^{\star}+\pi^{\star}\right) .
\end{aligned}
$$

The natural CFL condition is given by

$$
\frac{\Delta t}{\Delta x} \sigma^{\max } \leq \frac{1}{2},
$$

where $\sigma^{\max }=\max \left(\left|\sigma^{L}\right|,\left|\sigma^{*}\right|,\left|\sigma^{R}\right|\right)$.

\subsection{Properties of the scheme}

In this subsection we prove that our relaxation scheme is positive and satisfies a fully discrete entropy inequality, under some subcharacteristic conditions on the numerical relaxation parameters $a^{L}, a^{R}, b^{L}, b^{R}$. We begin with the entropy inequality, which is the strongest property. Let us recall that since our Godunov-type scheme is associated with an approximate Riemann solver of the original system (given by the exact Riemann solver of the relaxation system, restricted to the variables $\rho, \rho u$ ), one can use the Harten, Lax and van Leer formalism [31] and focus on this approximate Riemann solution, for which there is characterization of the entropy inequality, see also [4].

\subsubsection{Discrete entropy inequality}

Proposition 1. Let us assume that the intermediate densities $\rho^{L}, \rho^{R}, \rho^{L \star}$ and $\rho^{R \star}$ are positive. Then the approximate Riemann solver satisfies a discrete entropy inequality, i.e.

$$
\begin{gathered}
G\left(U^{R}\right)-G\left(U^{L}\right) \leq \sigma^{L}\left(\eta\left(U^{L \star}\right)-\eta\left(U^{L}\right)\right)+\sigma^{\star}\left(\eta\left(U^{R \star}\right)-\eta\left(U^{L \star}\right)\right) \\
+\sigma^{R}\left(\eta\left(U^{R}\right)-\eta\left(U^{R \star}\right)\right),
\end{gathered}
$$

provided that $a^{\alpha}, b^{\alpha}$ with $\alpha=L, R$ satisfy

$$
b^{\alpha} \leq a^{\alpha},
$$

and

$$
\left(\rho^{2} p^{\prime}\right)_{*}^{\alpha} \leq a^{\alpha} b^{\alpha}
$$


where $\left(\rho^{2} p^{\prime}\right)_{\star}^{\alpha}$ is defined as

$$
\left(\rho^{2} p^{\prime}\right)_{*}^{\alpha}=\sup _{\rho \in\left[\rho^{\alpha}, \rho^{\alpha \star}\right]} \rho^{2} p^{\prime}(\rho) .
$$

Observe that the entropy inequality is given under the assumption that the intermediate densities $\rho^{L \star}$ and $\rho^{R \star}$ are positive, which will be proved in the next paragraph. Notice also that $(2.39),(2.40)$ are discrete versions of the subcharacteristic conditions 2.29 associated with the diffusive asymptotics.

Proof. Let us recall that the positivity of the intermediate densities $\rho^{L \star}$ and $\rho^{R \star}$ is equivalent to $\sigma^{L}<\sigma^{\star}<\sigma^{R}$ (see the last lines of Subsection 2.4. Let us introduce a transported quantity $\mathcal{T}$,

$$
\partial_{t} \mathcal{T}+v \partial_{x} \mathcal{T}=0, \quad \text { initialized as } \mathcal{T}_{i}^{n}=1 / \rho_{i}^{n} .
$$

It completes the relaxation system (2.8)-(2.12) (without right-hand side). Since by 2.21) and 2.12 one has $\left(\partial_{t}+v \partial_{x}\right)(\pi+a b / \rho)=0$, the definition 2.42) implies that

$$
p\left(\frac{1}{\mathcal{T}}\right)+a b \mathcal{T}=\pi+\frac{a b}{\rho} .
$$

Let us now define the relaxation entropy $\Sigma$ by

$$
\Sigma(\rho, u, v, \pi, a, b, \mathcal{T})=\frac{\rho u^{2}}{2}+\rho \widehat{e}+\rho \frac{(v-u)^{2}}{2(a / b-1)},
$$

with

$$
\widehat{e}=e\left(\frac{1}{\mathcal{T}}\right)+\frac{\pi^{2}-p(1 / \mathcal{T})^{2}}{2 a b} .
$$

In 2.44) we use (2.39) with the convention that the last term vanishes if $b=a$ (in this case one has $v=u$ ). The definition 2.45 of $\widehat{e}$ is equivalent to

$$
\partial_{t}\left(\widehat{e}-\frac{\pi^{2}}{2 a b}\right)+v \partial_{x}\left(\widehat{e}-\frac{\pi^{2}}{2 a b}\right)=0, \quad \text { initialized as } \widehat{e}_{i}^{n}=e\left(\rho_{i}^{n}\right) .
$$

The relaxation entropy flux $G^{r}$ is defined by

$$
G^{r}=(\Sigma+\pi) v
$$

Notice that $\Sigma$ and $G^{r}$ and such that they coincide with the energy $\eta(U)$ and the energy flux $G(U)$ when $U^{r}$ is taken at equilibrium $\pi=p(\rho), v=u$, $\mathcal{T}=1 / \rho$. Let us now prove that $\partial_{t} \Sigma+\partial_{x} G^{r}=0$. From (2.16), 2.18) one gets

$$
\partial_{t} \frac{u^{2}}{2}+v \partial_{x} \frac{u^{2}}{2}+\frac{u}{\rho} \partial_{x} \pi=0, \quad \partial_{t} \frac{\pi^{2}}{2}+v \partial_{x} \frac{\pi^{2}}{2}+\frac{a b}{\rho} \pi \partial_{x} v=0 .
$$


Using (2.17) and (2.16) we have

$$
\partial_{t}(v-u)+v \partial_{x}(v-u)+\left(\frac{a}{b}-1\right) \frac{1}{\rho} \partial_{x} \pi=0,
$$

hence

$$
\partial_{t} \frac{(v-u)^{2}}{2}+v \partial_{x} \frac{(v-u)^{2}}{2}+(v-u)\left(\frac{a}{b}-1\right) \frac{1}{\rho} \partial_{x} \pi=0 .
$$

Summing 2.48 and 2.50 we get

$$
\begin{aligned}
\partial_{t}\left(\frac{u^{2}}{2}+\right. & \left.\frac{\pi^{2}}{2 a b}+\frac{1}{a / b-1} \frac{(v-u)^{2}}{2}\right) \\
& +v \partial_{x}\left(\frac{u^{2}}{2}+\frac{\pi^{2}}{2 a b}+\frac{1}{a / b-1} \frac{(v-u)^{2}}{2}\right)+\frac{1}{\rho} \partial_{x}(\pi v)=0 .
\end{aligned}
$$

Adding also (2.46) and multiplying the result by $\rho$ using (2.8), we obtain

$\partial_{t}\left(\frac{\rho u^{2}}{2}+\rho \widehat{e}+\frac{\rho}{a / b-1} \frac{(v-u)^{2}}{2}\right)+\partial_{x}\left(\left(\frac{\rho u^{2}}{2}+\rho \widehat{e}+\frac{\rho}{a / b-1} \frac{(v-u)^{2}}{2}+\pi\right) v\right)=0$

which is the claim $\partial_{t} \Sigma+\partial_{x} G^{r}=0$. Using then that in a system with all linearly degenerate eigenvalues, any formally conservative equation is indeed satisfied in the sense of distributions, the conservative equation 2.52 implies the Rankine-Hugoniot relations

$$
\begin{gathered}
G\left(U^{R}\right)-G^{r}\left(U^{r, R \star}\right)=\sigma^{R}\left(\eta\left(U^{R}\right)-\Sigma\left(U^{r, R \star}\right)\right), \\
G^{r}\left(U^{r, R \star}\right)-G^{r}\left(U^{r, L \star}\right)=\sigma^{\star}\left(\Sigma\left(U^{r, R \star}\right)-\Sigma\left(U^{r, L \star}\right)\right), \\
G^{r}\left(U^{r, L \star}\right)-G\left(U^{L}\right)=\sigma^{L}\left(\Sigma\left(U^{r, L \star}\right)-\eta\left(U^{L}\right)\right) .
\end{gathered}
$$

These relations can also be checked directly with the explicit values of the intermediate states given by (2.31). We are now going to prove the inequalities

$$
\begin{aligned}
& \eta\left(U^{R \star}\right) \leq \Sigma\left(U^{r, R \star}\right), \\
& \eta\left(U^{L \star}\right) \leq \Sigma\left(U^{r, L \star}\right) .
\end{aligned}
$$

Indeed, summing up the three lines of 2.53) and using (2.54) (and the property that $\sigma^{R}-\sigma^{\star} \geq 0, \sigma^{\star}-\sigma^{L} \geq 0$ ) resumes to the claimed inequality 2.38). Now, to prove 2.54), observe that these inequalities express that the entropy of the original system, which coincides with the relaxation entropy taken at equilibrium, is smaller than the relaxation entropy. By definition of $\Sigma, 2.54$ also writes

$$
\begin{aligned}
& e\left(\rho^{R \star}\right)-e\left(1 / \mathcal{T}^{R \star}\right) \leq \frac{\left(\pi^{R \star}\right)^{2}-p\left(1 / \mathcal{T}^{R \star}\right)^{2}}{2 a^{R} b^{R}}+\frac{\left(v^{R \star}-u^{R \star}\right)^{2}}{2\left(a^{R} / b^{R}-1\right)}, \\
& e\left(\rho^{L \star}\right)-e\left(1 / \mathcal{T}^{L \star}\right) \leq \frac{\left(\pi^{L \star}\right)^{2}-p\left(1 / \mathcal{T}^{L \star}\right)^{2}}{2 a^{L} b^{L}}+\frac{\left(v^{L \star}-u^{L \star}\right)^{2}}{2\left(a^{L} / b^{L}-1\right)} .
\end{aligned}
$$


Since by 2.43 $\pi=p(1 / \mathcal{T})+a b(\mathcal{T}-1 / \rho)$, neglecting the last term with (2.39), it is sufficient to prove that

$$
\begin{aligned}
& e\left(\rho^{R \star}\right)-e\left(1 / \mathcal{T}^{R \star}\right) \leq \frac{a^{R} b^{R}}{2}\left(\mathcal{T}^{R \star}-1 / \rho^{R \star}\right)^{2}+p\left(1 / \mathcal{T}^{R \star}\right)\left(\mathcal{T}^{R \star}-1 / \rho^{R \star}\right), \\
& e\left(\rho^{L \star}\right)-e\left(1 / \mathcal{T}^{L \star}\right) \leq \frac{a^{L} b^{L}}{2}\left(\mathcal{T}^{L \star}-1 / \rho^{L \star}\right)^{2}+p\left(1 / \mathcal{T}^{L \star}\right)\left(\mathcal{T}^{L \star}-1 / \rho^{L \star}\right) .
\end{aligned}
$$

For given $\rho^{*}, a, b$ consider now the function

$$
\varphi(\rho)=e(\rho)-e\left(\rho^{*}\right)+\frac{a b}{2}\left(1 / \rho-1 / \rho^{*}\right)^{2}+p(\rho)\left(1 / \rho-1 / \rho^{*}\right) .
$$

Then using (2.6),

$$
\varphi^{\prime}(\rho)=\left(p^{\prime}(\rho)-\frac{a b}{\rho^{2}}\right)\left(1 / \rho-1 / \rho^{*}\right) .
$$

We deduce that if there is an interval $I \ni \rho^{*}$ such that

$$
\sup _{\rho \in I} \rho^{2} p^{\prime}(\rho) \leq a b,
$$

then $\varphi^{\prime}(\rho)$ has the same sign as $\rho-\rho^{*}$ over $I$, and thus $\varphi(\rho) \geq 0$ for all $\rho \in I$. We deduce that the first inequality in (2.56) holds provided that $(2.59)$ holds with $a=a^{R}, b=b^{R}, I=\left[\rho^{R *}, 1 / \mathcal{T}^{R \star}\right]$ (take $\left.\rho^{*}=\rho^{R *}, \rho=1 / \mathcal{T}^{R \star}\right)$. Similarly the second inequality in (2.56) holds provided that 2.59 holds with $a=a^{L}, b=b^{L}, I=\left[\rho^{L *}, 1 / \mathcal{T}^{L \star}\right]$. But according to 2.42 ,

$$
\mathcal{T}^{L}=\mathcal{T}^{L \star}=1 / \rho^{L}, \quad \mathcal{T}^{R}=\mathcal{T}^{R \star}=1 / \rho^{R},
$$

and the two conditions reduce to (2.40, 2.41). This finishes the proof of (2.54).

\subsubsection{Entropy inequality and steady solutions}

In this subsection we show how the validity of a discrete entropy inequality can be used to show that steady periodic solutions of the underlying scheme are also constant in space. This property is of specific interest in the context of the next section devoted to the low Mach number flows, when evoking the so-called checkerboard modes.

Let us first recall that thanks to the Harten, Lax and Van Leer formalism [31] and the validity of Proposition 1, the fully discrete entropy inequality also writes

$$
\begin{aligned}
& \eta\left(U_{i}^{n+1}\right)-\eta\left(U_{i}^{n}\right)+\frac{\Delta t}{\Delta x}\left(G_{i+1 / 2}^{n}-G_{i-1 / 2}^{n}\right) \\
= & \eta\left(U_{i}^{n+1}\right)-\frac{1}{\Delta x} \int_{x_{i-1 / 2}}^{x_{i+1 / 2}} \Sigma\left(U^{r}\left(t^{n+1}, x\right)\right) d x \leq 0,
\end{aligned}
$$


with

$$
G_{i+1 / 2}^{n}=G\left(U_{i}^{n}, U_{i+1}^{n}\right)
$$

and the notations of the previous paragraph,

$$
G\left(U^{L}, U^{R}\right)= \begin{cases}G\left(U^{L}\right) \text { if } \sigma^{L} \geq 0, \\ G^{r}\left(U^{r, L \star}\right)=\left(\Sigma^{L \star}+\pi^{\star}\right) v^{\star} & \text { if } \sigma^{L} \leq 0 \leq \sigma^{*}, \\ G^{r}\left(U^{r, R \star}\right)=\left(\Sigma^{R \star}+\pi^{\star}\right) v^{\star} & \text { if } \sigma^{*} \leq 0 \leq \sigma^{R}, \\ G\left(U^{R}\right) \text { if } \sigma^{R} \leq 0 . & \end{cases}
$$

Indeed, the equality in 2.61) comes from integrating 2.52 in time and space, while the inequality follows from

$$
\eta\left(U_{i}^{n+1}\right) \leq \frac{1}{\Delta x} \int_{x_{i-1 / 2}}^{x_{i+1 / 2}} \eta\left(U\left(t^{n+1}, x\right)\right) d x \leq \frac{1}{\Delta x} \int_{x_{i-1 / 2}}^{x_{i+1 / 2}} \Sigma\left(U^{r}\left(t^{n+1}, x\right)\right) d x,
$$

where $U(t, x)$ stands for the approximate Riemann solution, i.e. the conservative part of $U^{r}(t, x)$, the first inequality is Jensen's whereas the second one is indeed (2.54). Let us set

$$
D_{i}^{n}=\eta\left(U_{i}^{n+1}\right)-\eta\left(U_{i}^{n}\right)+\frac{\Delta t}{\Delta x}\left(G_{i+1 / 2}^{n}-G_{i-1 / 2}^{n}\right) .
$$

Considering a steady and space periodic solution, it comes

$$
\sum_{i} D_{i}^{n}=0
$$

Since $D_{i}^{n} \leq 0$ for all $i$, we get that $D_{i}^{n}=0$ for all $i$, or equivalently equality in both inequalities of 2.64). In particular we have $\eta\left(U\left(t^{n+1}, x\right)\right)=$ $\Sigma\left(U^{r}\left(t^{n+1}, x\right)\right)$ for all $x$ in the cell $\kappa_{i}$ and for all $i$. According to the analysis of the inequalities (2.54) provided in the proof of Proposition 1, this implies that $v=u$ and $\mathcal{T}=1 / \rho$. Then (2.43) yields $\pi=p(\rho)$. This means that we are at equilibrium for all $x$ in all cells $\kappa_{i}$. Focusing ourselves on a local Riemann problem, it means that $\mathcal{T}^{L \star}=1 / \rho^{L \star}, \mathcal{T}^{R \star}=1 / \rho^{R \star}$, $v^{L \star}=u^{L \star}, v^{R \star}=u^{R \star}, \pi^{L \star}=p\left(\rho^{L \star}\right), \pi^{R \star}=p\left(\rho^{R \star}\right)$. But since $\pi^{L \star}=\pi^{R \star}$ and using (2.60) we get that $\rho^{L \star}=\rho^{R \star}=\rho^{L}=\rho^{R}$. We have hence $\pi^{\star}=p\left(\rho^{L}\right)=p\left(\rho^{R}\right)$, thus with the explicit value of $\pi^{\star}$ given in 2.31) we get $v^{L}=v^{R}$ i.e. $u^{L}=u^{R}$. Thus $U^{L}=U^{R}$ for all local Riemann problems, which means that the solution is constant in space.

\subsubsection{Practical definitions of $a$ and $b$}

The stability conditions (2.39), 2.40, (2.41) do not provide us with an explicit definition of $a^{\alpha}$ and $b^{\alpha}, \alpha=L, R$ because the intermediate states $\rho^{\alpha \star}$ actually depend on $a^{\alpha}$ and $b^{\alpha}, \alpha=L, R$, as seen on (2.31). In this 
subsection we give explicit sufficient inequalities on $a^{\alpha}$ and $b^{\alpha}$ following the ideas introduced in [8].

Let us first recall that by assumption the pressure law verifies 2.2 and (2.3) for some constant $\beta>1$. We summarize our results in the following theorem.

Theorem 1. Under the assumptions (2.2), (2.3) on the pressure law, consider the two-speed approximate Riemann solver with intermediate values and speeds defined by (2.31), (2.32) with the initial data at equilibrium $\pi^{L}=p\left(\rho^{L}\right), \pi^{R}=p\left(\rho^{R}\right), v^{L}=u^{L}, v^{R}=u^{R}$. Its numerical flux is given by (2.35), 2.36). Assume that $a^{L}, a^{R}, b^{L}, b^{R}$ are defined in some way, depending on the left/right states $U^{L}, U^{R}$, so that they satisfy

$$
\begin{gathered}
a^{L} \geq b^{L}, \quad a^{R} \geq b^{R}, \\
\frac{b^{L}}{\rho^{L}} \geq a_{q}^{L}, \quad \frac{b^{R}}{\rho^{R}} \geq a_{q}^{R}, \\
\frac{\sqrt{a^{L} b^{L}}}{\rho^{L}} \geq \sqrt{p^{L}}\left(1+\beta X^{L}\right), \quad \frac{\sqrt{a^{R} b^{R}}}{\rho^{R}} \geq \sqrt{p^{\prime R}}\left(1+\beta X^{R}\right),
\end{gathered}
$$

for some $a_{q}^{L}, a_{q}^{R}$ (depending also on $U^{L}, U^{R}$ ), with $p^{L}=p^{\prime}\left(\rho^{L}\right), p^{R}=$ $p^{\prime}\left(\rho^{R}\right)$, and $X^{L}, X^{R}$ defined by 2.71, 2.72. Then the solver satisfies the following properties.

(i) It is conservative, and consistent with (2.1),

(ii) It is positive in density $\rho$, and the ordering of the speeds (2.30) is valid, (iii) It satisfies a discrete entropy inequality with numerical entropy flux given by (2.63).

Therefore, the scheme (2.34) inherits these properties under the CFL condition 2.37.

Proof. Let us first remark that 2.65 implies that $\sqrt{a^{L} b^{L}} \leq a^{L}, \sqrt{a^{R} b^{R}} \leq$ $a^{R}$, thus with 2.67) we obtain

$$
\frac{a^{L}}{\rho^{L}} \geq \sqrt{p^{L}}\left(1+\beta X^{L}\right), \quad \frac{a^{R}}{\rho^{R}} \geq \sqrt{p^{R}}\left(1+\beta X^{R}\right) .
$$

The fact that we have an approximate Riemann solver is obvious by construction and according to the general theory of relaxation solvers [4]. The property (i) also follows from this general theory, as well as the conclusion on the scheme under the CFL condition (2.37). Thus it remains to prove (ii) and (iii). The equivalence between the positivity of density and the ordering of the speeds has already been established (last lines of Subsection 2.4). Thus it remains to prove the positivity of the intermediate densities, and the entropy condition. The latter is obtained via Proposition 1 . We 
begin by estimating the intermediate densities from 2.31,

$$
\begin{aligned}
\frac{1}{\rho^{L *}} & =\frac{1}{\rho^{L}}+\frac{b^{R}\left(v^{R}-v^{L}\right)+\pi^{L}-\pi^{R}}{a^{L}\left(b^{L}+b^{R}\right)} \\
& \geq \frac{1}{\rho^{L}}-\frac{b^{R}\left(v^{L}-v^{R}\right)_{+}}{a^{L}\left(b^{L}+b^{R}\right)}-\frac{\left(\pi^{R}-\pi^{L}\right)_{+}}{a^{L}\left(b^{L}+b^{R}\right)} \\
& \geq \frac{1}{\rho^{L}}-\frac{\left(v^{L}-v^{R}\right)_{+}}{a^{L}}-\frac{\left(\pi^{R}-\pi^{L}\right)_{+}}{a^{L}\left(\rho^{L} a_{q}^{L}+\rho^{R} a_{q}^{R}\right)},
\end{aligned}
$$

and similarly

$$
\begin{aligned}
\frac{1}{\rho^{R *}} & =\frac{1}{\rho^{R}}+\frac{b^{L}\left(v^{R}-v^{L}\right)+\pi^{R}-\pi^{L}}{a^{R}\left(b^{L}+b^{R}\right)} \\
& \geq \frac{1}{\rho^{R}}-\frac{b^{L}\left(v^{L}-v^{R}\right)_{+}}{a^{R}\left(b^{L}+b^{R}\right)}-\frac{\left(\pi^{L}-\pi^{R}\right)_{+}}{a^{R}\left(b^{L}+b^{R}\right)} \\
& \geq \frac{1}{\rho^{R}}-\frac{\left(v^{L}-v^{R}\right)_{+}}{a^{R}}-\frac{\left(\pi^{L}-\pi^{R}\right)_{+}}{a^{R}\left(\rho^{L} a_{q}^{L}+\rho^{R} a_{q}^{R}\right)},
\end{aligned}
$$

where we have used 2.66). Let us now define

$$
\begin{aligned}
& X^{L}=\frac{1}{\sqrt{p^{L}}}\left(\left(v^{L}-v^{R}\right)_{+}+\frac{\left(\pi^{R}-\pi^{L}\right)_{+}}{\rho^{L} a_{q}^{L}+\rho^{R} a_{q}^{R}}\right), \\
& X^{R}=\frac{1}{\sqrt{p^{\prime}}}\left(\left(v^{L}-v^{R}\right)_{+}+\frac{\left(\pi^{L}-\pi^{R}\right)_{+}}{\rho^{L} a_{q}^{L}+\rho^{R} a_{q}^{R}}\right),
\end{aligned}
$$

so that $(2.69)$ and $(2.70)$ write

$$
\frac{1}{\rho^{L *}} \geq \frac{1}{\rho^{L}}\left(1-\frac{\rho^{L} \sqrt{p^{\prime L}}}{a^{L}} X^{L}\right), \quad \frac{1}{\rho^{R *}} \geq \frac{1}{\rho^{R}}\left(1-\frac{\rho^{R} \sqrt{p^{\prime}}}{a^{R}} X^{R}\right) .
$$

With 2.68) we thus obtain

$$
\frac{1}{\rho^{L *}} \geq \frac{1}{\rho^{L}}\left(1-\frac{X^{L}}{1+\beta X^{L}}\right), \quad \frac{1}{\rho^{R *}} \geq \frac{1}{\rho^{R}}\left(1-\frac{X^{R}}{1+\beta X^{R}}\right) .
$$

We deduce that $\rho^{L *}>0, \rho^{R *}>0$ and

$$
0<\rho^{L *} \leq \rho^{L} / x^{L}, \quad 0<\rho^{R *} \leq \rho^{R} / x^{R},
$$

with (recall that we assume that $\beta>1$ )

$$
x^{L}=1-\frac{X^{L}}{1+\beta X^{L}} \quad \text { and } \quad x^{R}=1-\frac{X^{R}}{1+\beta X^{R}} \quad \in\left(\frac{\beta-1}{\beta}, 1\right] .
$$


Since we have the assumptions 2.2 , 2.3 on the pressure law, we can apply Lemma 3.1 in [8]. It gives that

$\frac{\rho^{L}}{x^{L}} \sqrt{p^{\prime}\left(\frac{\rho^{L}}{x^{L}}\right)} \leq \rho^{L} \sqrt{p^{\prime}\left(\rho^{L}\right)}\left(1+\beta X^{L}\right), \quad \frac{\rho^{R}}{x^{R}} \sqrt{p^{\prime}\left(\frac{\rho^{R}}{x^{R}}\right)} \leq \rho^{R} \sqrt{p^{\prime}\left(\rho^{R}\right)}\left(1+\beta X^{R}\right)$.

According to the monotonicity of $\rho \sqrt{p^{\prime}}$ stated in $(2.2)$, this estimate together with 2.75 allow then to estimate the supremum, namely

$$
\begin{gathered}
\sup _{\rho \in\left[\rho^{L}, \rho^{L *}\right]} \rho \sqrt{p^{\prime}(\rho)} \leq \rho^{L} \sqrt{p^{\prime}}\left(1+\beta X^{L}\right), \\
\sup _{\rho \in\left[\rho^{R}, \rho^{R *}\right]} \rho \sqrt{p^{\prime}(\rho)} \leq \rho^{R} \sqrt{p^{\prime}}\left(1+\beta X^{R}\right) .
\end{gathered}
$$

To conclude we apply Proposition 1 . The condition $(2.39)$ is just (2.65), and the conditions (2.40), 2.41) follow from 2.77), 2.78), (2.67).

Some comments on the stability conditions (2.65), (2.66), 2.67) are in order. They are of great practical usefulness because one can satisfy them easily while imposing some particular scales in the solver. The most simple example is the "classical one" $a=b$ for which one chooses

$$
a_{q}^{L}=\sqrt{p^{L}}, \quad a_{q}^{R}=\sqrt{p^{\prime}}
$$

and

$$
\frac{a^{L}}{\rho^{L}}=\frac{b^{L}}{\rho^{L}}=\sqrt{p^{L}}\left(1+\beta X^{L}\right), \quad \frac{a^{R}}{\rho^{R}}=\frac{b^{R}}{\rho^{R}}=\sqrt{p^{\prime R}}\left(1+\beta X^{R}\right),
$$

with $X^{L}, X^{R}$ defined by (2.71), 2.72). In these formulas we see the advantage of taking different values for $a^{L}, a^{R}$ : they are adapted to the magnitude of the density on each side. In particular, we see that if for example $\rho^{L} \rightarrow 0$ while $\rho^{R}>0$ (Riemann problem with vacuum on one side), the ratios $a^{L} / \rho^{L}$ and $a^{R} / \rho^{R}$ do not blow up, and the CFL condition (2.37) remains finite knowing (2.32) (what would not be the case with a choice $a^{L}=a^{R}$ ).

When making a "non classical choice", as in low Mach situations, one has to see that $a$ has to be large enough according to (2.65), 2.67) to ensure stability, but $b$ can remain bounded (for example $b / \rho=a_{q}$ ). Looking at the viscosity matrix (2.28) associated to the scheme, it means that the numerical viscosity on the density variable $\rho$ becomes large, but the numerical viscosity on the velocity $u$ remains bounded.

\subsection{A numerical illustration}

At this stage, our two-speed numerical scheme is just another finite volume scheme which satisfies positivity and entropy inequality properties. In this 
part, our objective is not to consider many test cases and to report on the behaviour of this new scheme. Indeed and as clearly expected, it actually provides us with numerical solutions with standard accuracy. On the contrary, we show only one numerical test in order to give an insight into the influence of choosing the wave speeds $a$ and $b$. This test case is taken from [22, 27]. The pressure law is $p(\rho)=\rho^{2}$, and the initial condition writes

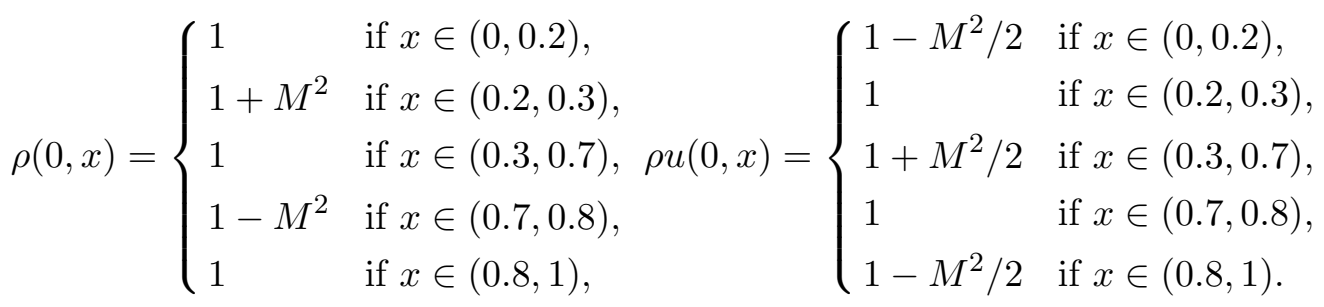

The spatial domain is $(0,1)$, periodic boundary conditions are applied and we set $M^{2}=0.99$. In Figures (2), (3), the density profile (left) and momentum profile (right) are displayed at initial time and at time $t=0.051$, with a spacestep of $\Delta x=1 / 300$ for our two-speed relaxation scheme. We have set $a_{q}=\sqrt{p^{\prime}}, b / \rho=\sqrt{p^{\prime}}(1+\beta X)$, and $a=b$ (i.e. the classical choice (2.79), (2.80), in blue), $a=2 b$ (in yellow) and $a=4 b$ (in red). The reference solution (black) is computed using a very fine mesh given by $\Delta x=1 / 3000$. The numerical test case shows that the choice $a=b$ gives the best result and seems optimal. Indeed it gives the "smallest" viscosity matrix (2.28). However, it will be seen that the opportunity of choosing different $a$ and $b$ is relevant for the study of low Mach number regimes. This is the aim of the next section.

\section{Application to low Mach number flows}

In this section we apply our two-speed relaxation solver to low Mach flows. When the Mach number is small a well-known difficulty is to design asymptoticpreserving schemes, which means schemes that are uniformly accurate with respect to the Mach number. It has been pointed out in [7] that until now there was no asymptotic-preserving scheme which satisfies a fully discrete entropy inequality and which is first-order accurate in the low Mach number regime. Indeed a scheme proposed in [7] is entropy-satisfying and asymptotic-preserving but the order of accuracy is only $2 / 3$ in the incompressible asymptotics. On the contrary, the Lagrange-Projection scheme proposed in [14] (see also [15]) is first-order accurate (uniformly with respect to the Mach number) but the underlying entropy inequality is no longer valid when we are too close to the asymptotic limit. We show here that the two-speed relaxation solver with adequate choice of the speeds yields a scheme satisfying all the above properties: asymptotic-preserving with uni- 

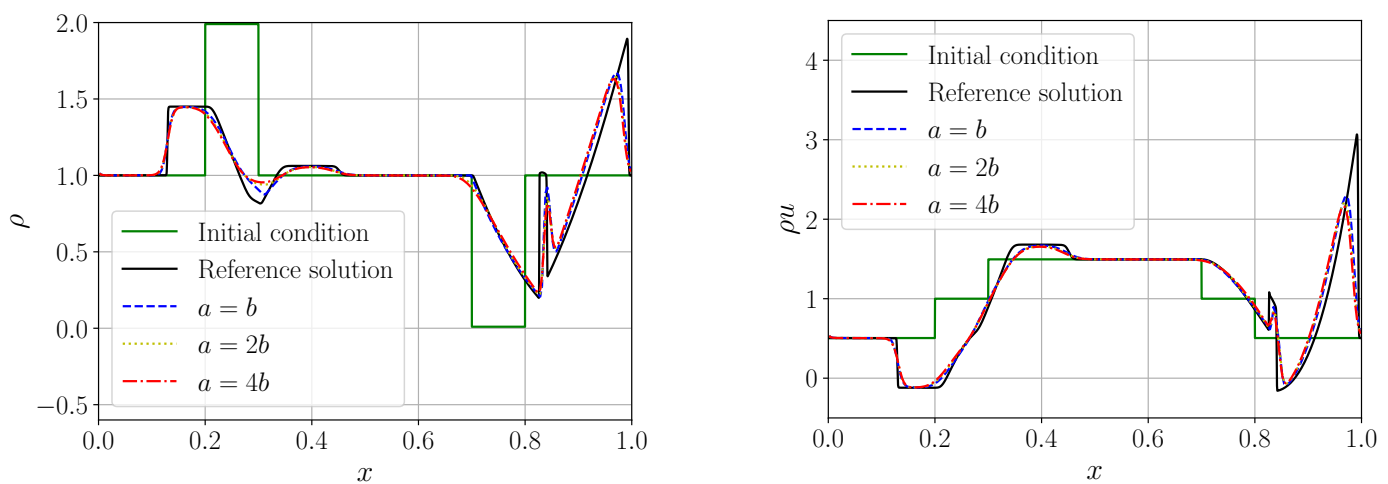

Figure 2: Density (left) and momentum (right) at time $t=0.051$ in the case $a=b$ (blue), $a=2 b$ (yellow) and $a=4 b$ (red).
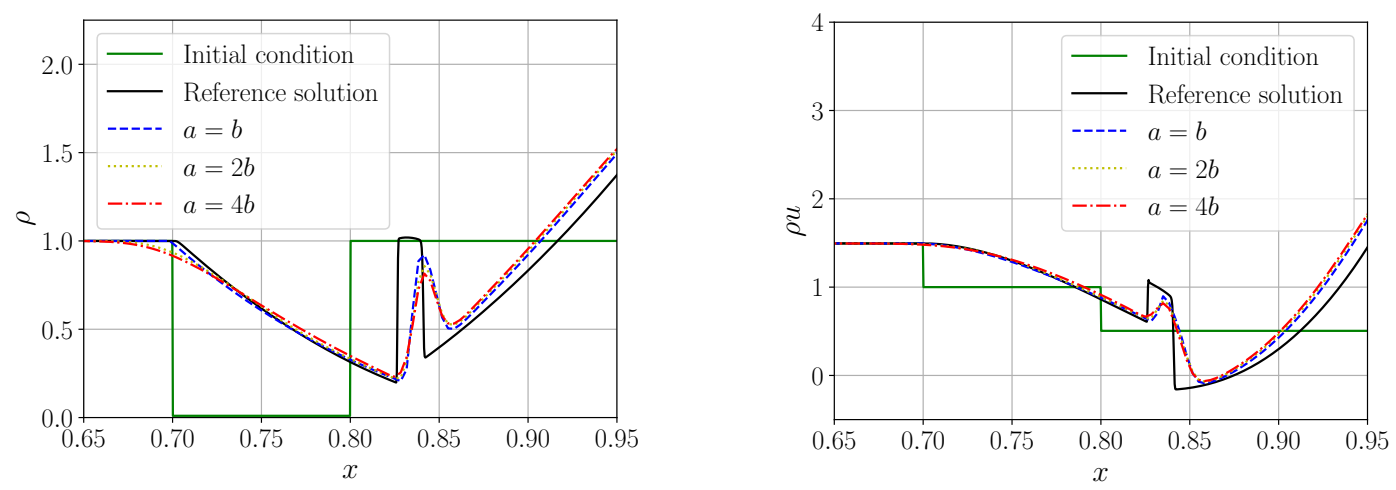

Figure 3: Zoom on the density (left) and momentum (right) curves.

form first-order accuracy, and entropy inequality. The entropy inequality ensures strong stability properties, and prevents any unphysical growth of the numerical approximation.

Let us consider the dimensionless Euler equations in several space dimensions which write with classical notations

$$
\partial_{t}\left(\begin{array}{c}
\rho \\
\rho u
\end{array}\right)+\operatorname{div}_{x}\left(\begin{array}{c}
\rho u \\
\rho u \otimes u+\frac{\bar{p}(\rho)}{M^{2}} I d
\end{array}\right)=0,
$$

where $M$ denotes the so-called Mach number. It means that the pressure law is now

$$
p(\rho)=\frac{\bar{p}(\rho)}{M^{2}},
$$

where $M$ is an eventually small parameter. In several space dimensions we can apply our 1d solver at each interface between two cells, in a classical finite volume fashion. The transverse velocity is solved as usual by a passive 
transport scheme. We can observe that then there is no special $2 \mathrm{~d}$ treatment in this algorithm, it is only the very standard approach. Moreover it is fully explicit, although a time implicit algorithm like in [12] could be considered.

\subsection{The low Mach number regime}

Let us briefly recall the incompressible asymptotics of (3.1) when the Mach number $M$ goes to zero. We consider the following expansions in powers of $M$,

$$
\begin{aligned}
& \rho=\rho^{0}+M \rho^{1}+M^{2} \rho^{2}+\mathcal{O}\left(M^{3}\right), \\
& u=u^{0}+M u^{1}+M^{2} u^{2}+\mathcal{O}\left(M^{3}\right),
\end{aligned}
$$

where $\rho^{i}, u^{i}, i=0, \ldots, 2$ are functions of time and space. It gives in particular

$$
\bar{p}=\bar{p}^{0}+M \bar{p}^{1}+M^{2} \bar{p}^{2}+\mathcal{O}\left(M^{3}\right)=\bar{p}\left(\rho^{0}\right)+M \bar{p}^{\prime}\left(\rho^{0}\right) \rho^{1}+\mathcal{O}\left(M^{2}\right) .
$$

The second equation of (3.1) gives that $\nabla_{x}\left(\bar{p}(\rho) / M^{2}\right)$ is bounded independently of $M$. Therefore, $\nabla_{x} \rho^{0}=0$ and $\nabla_{x} \rho^{1}=0$. It follows that

$$
\rho^{0}(t, x)=\rho^{0}(t) \quad \text { and } \quad \rho^{1}(t, x)=\rho^{1}(t) .
$$

In particular we get the limit of (3.1) as $M \rightarrow 0$,

$$
\left\{\begin{array}{l}
\partial_{t} \rho^{0}+\operatorname{div}_{x}\left(\rho^{0} u^{0}\right)=0, \\
\partial_{t}\left(\rho^{0} u^{0}\right)+\operatorname{div}_{x}\left(\rho^{0} u^{0} \otimes u^{0}+\bar{p}^{2} I d\right)=0 .
\end{array}\right.
$$

Assuming the velocity boundary conditions

$$
\int_{\Omega} \operatorname{div}_{x}(u) d x=0 \text { i.e. } \int_{\partial \Omega} u . n d \sigma=0
$$

and integrating in space the first equation of (3.4) yields $\partial_{t} \rho^{0}=0$, so that $\rho^{0}$ is constant in both space and time. Therefore we recover the incompressibility conditions

$$
\rho^{0}=c s t, \quad \operatorname{div}_{x} u^{0}=0,
$$

and the evolution of $u^{0}$ is given by

$$
\partial_{t} u^{0}+\operatorname{div}_{x}\left(u^{0} \otimes u^{0}+\frac{\bar{p}^{2}}{\rho^{0}} I d\right)=0 .
$$

The system (3.6), (3.7), where the value of $\rho^{0}$ can be eliminated, is the well-known Euler system for incompressible fluids. Doing again the above argument with the boundary condition (3.5) using the expansion (3.3) of $\rho$ gives also that $\rho^{1}=c s t$. To simplify we shall assume that there is no constant fluctuation in $\rho$, that is

$$
\rho^{1}=0
$$


(this depending on initial conditions). It implies that

$$
\rho=\rho^{0}+\mathcal{O}\left(M^{2}\right),
$$

and thus that (3.1) can be written (for smooth solutions)

$$
\begin{aligned}
& \operatorname{div}_{x} u=\mathcal{O}\left(M^{2}\right), \\
& \partial_{t} u+\operatorname{div}_{x} u \otimes u+\nabla_{x}\left(\frac{\bar{p}(\rho)}{\rho^{0} M^{2}}\right)=\mathcal{O}\left(M^{2}\right),
\end{aligned}
$$

which means that the limit is achieved up to errors of the order of $M^{2}$.

When considering a numerical method for (3.1), the consistency of the scheme with the limit model when the Mach number $M$ goes to zero is an important property. A stronger property is the asymptotic-preserving property, which means uniform accuracy with respect to $M$.

\subsection{Definition of the relaxation speeds for the low Mach regime}

We propose now practical definitions of the wave speeds $a^{L}, a^{R}, b^{L}, b^{R}$, in agreement with the analysis proposed in Subsection 2.6.3. In order to fulfill the stability conditions (2.65), (2.66), 2.67), we take for $\alpha=L, R$ (recall that the pressure $p$ is related to $\bar{p}$ by $(3.2)$ )

$$
\begin{aligned}
a_{q}^{\alpha} & =\min (1, M) \sqrt{\frac{\bar{p}^{\prime \alpha}}{M^{2}}}, \\
\frac{b^{\alpha}}{\rho^{\alpha}} & =\min (1, M) \sqrt{\frac{\bar{p}^{\prime \alpha}}{M^{2}}}\left(1+\beta X^{\alpha}\right), \\
\frac{a^{\alpha}}{\rho^{\alpha}} & =\frac{1}{\min (1, M)} \sqrt{\frac{\bar{p}^{\prime \alpha}}{M^{2}}}\left(1+\beta X^{\alpha}\right),
\end{aligned}
$$

where the $X^{\alpha}$ are defined by (2.71), 2.72). Observe that in the case $M \geq 1$, we recover the classical choice $a=b$, i.e. $(2.79),(2.80)$. In the general case, (3.11) differs from the classical choice only by the factor $\min (1, M)$ in the three formulas. When $M \rightarrow 0$, we observe that $a_{q}$ and $b$ remain bounded, whereas $a \sim 1 / M^{2}$.

Consider the two-speed relaxation scheme with the choice (3.11) of the wave speeds, where the pressure law takes the form (3.2), with still the assumptions (2.2), 2.3). Then the conclusions of Theorem 1 are valid at fixed $M$, but without limitation on the size of $M$ (in particular the density remains positive and the discrete entropy inequality holds), with the modification that in several space dimensions we apply the 1d solver at each cell interface in the finite volume fashion, and the CFL condition is modified by a factor depending on the geometry of the mesh. We highlight the importance of this result and point out that it also holds working with unstructured meshes. In addition we have the following properties. 
Theorem 2. The two-speed relaxation scheme with the choice (3.11) of the wave speeds is asymptotic-preserving in the low Mach number regime. More precisely, assuming that we are in the low Mach regime in the sense that for the considered initial data the space continuous limit explained in Subsection 3.1 is valid with $\rho=\rho^{0}+\mathcal{O}\left(M^{2}\right)$ and $\nabla_{x} p=\mathcal{O}(1)$ and the solution to the incompressible system (3.6), (3.7) is smooth, one has that

a) the scheme is first-order uniformly with respect to the Mach number $M$, b) for $M<\sqrt{k \Delta x}$ (with $k$ a constant), the scheme is consistent at first order with the incompressible limit model (3.6), (3.7). Moreover the scheme is consistent with the incompressibility equation (3.6) uniformly in time.

The properties $a$ ), $b$ ) do not follow from Theorem 1, they are proved in Subsection 3.3. Technically, we prove that in $1 \mathrm{~d}$ the scheme satisfies an expansion in terms of $M$ and $\Delta x$ both going to zero at arbitrary respective speeds,

$$
\begin{aligned}
& F\left(U^{L}, U^{R}\right)-\frac{F\left(U^{L}\right)+F\left(U^{R}\right)}{2} \\
=-\frac{\Delta x}{2} \sqrt{\bar{p}^{\prime}} \partial_{x}\left(\frac{\rho-\rho^{0}}{M^{2}}\right)(1, u)-\frac{\Delta x}{2}(\rho|u| & \left.+\rho \sqrt{\bar{p}^{\prime}}\right)\left(0, \partial_{x} u\right) \\
& +\mathcal{O}\left(\Delta x^{2}\right)+\mathcal{O}(M \Delta x),
\end{aligned}
$$

where $F\left(U^{L}, U^{R}\right)$ is the numerical flux, $U^{L}, U^{R}$ are the left and right values of $U$, the nonlinearity $F(U)$ comes from (2.1), and on the right-hand side of 3.12 the local values of $\rho, u$ are related to the exact solution to the compressible model (3.1).

Before going into the proof of Theorem 2, some comments are in order. First, our scheme is not defined for $M=0$, it would give infinite propagation speeds. Nevertheless, $M>0$ can be arbitrary small. The same remark is valid for $\Delta x$ : the scheme is defined only for $\Delta x>0$. Second, the consistency of a scheme is a property relative to $\Delta x$ tending to 0 , it is thus meaningless to speak of consistency "at fixed $\Delta x$ ". Therefore, when talking of consistency "as $M \rightarrow 0$ " it means necessarily that both $M \rightarrow 0$ and $\Delta x \rightarrow 0$. The real issue is what are the assumptions on the respective speeds of these two parameters going to 0 .

Property a) implies that if $M$ is arbitrary small the scheme is uniformly consistent with the barotropic system (3.1) (that involves the parameter $M$ ), which itself is close to the incompressible limit if $M$ is small enough. This implies that for $M \rightarrow 0$ (which has to be understood in the sense that $M$ and $\Delta x$ both tend to 0 , but $M$ is arbitrary smaller than $\Delta x$ ), the scheme becomes consistent with the incompressible limit. Next, property b) states that for $M<\sqrt{k \Delta x}$ (with $k$ a constant), the scheme is consistent at first order with the incompressible limit model. This condition $M<\sqrt{k \Delta x}$ is in particular satisfied when $M \rightarrow 0$, thus we get again that as $M \rightarrow 0$ the 
scheme becomes consistent with the incompressible model (limit-consistency of the scheme). Indeed this property b) slightly extends the limit-consistency property, for example it is possible to choose $\Delta x \sim M^{2}$ and still keep the consistency.

An interpretation of 3.12 is that in $1 \mathrm{~d}$ the scheme has an equivalent equation of the form (2.27) with a numerical viscosity matrix given by (2.28) in which we set formally

$$
\varepsilon\left(\frac{a}{b}-1\right) p^{\prime}=\frac{\Delta x}{2} \frac{\sqrt{\bar{p}^{\prime}}}{M^{2}}, \quad \varepsilon\left(\frac{a b}{\rho^{2}}-p^{\prime}\right)=\frac{\Delta x}{2}\left(|u|+\sqrt{\bar{p}^{\prime}}\right) .
$$

However these relations that formally identify the viscosity matrix (2.28) of the relaxation system and the numerical viscosity should in principle be corrected by the numerical viscosity of the exact Godunov scheme, that is not taken into account in (2.28) whereas it is included in the formulas (3.12). Notice also that according to [3, Proposition 3.1], the relevant value of $\varepsilon$ is $\varepsilon=\Delta t / 2$.

To compare with, if one considers the classical one-speed relaxation scheme, i.e. the choice 2.80 for which $b=a=\mathcal{O}(1 / M)$, then the numerical diffusion coefficients (excluding the factor $\Delta x$ ) behave as $\mathcal{O}(1 / M)$ for $\rho$ and $\mathcal{O}(1 / M)$ for $u$. In other words, the numerical diffusion on the mass equation is small (knowing (3.15)), while it is extremely large on the momentum equation. As a matter of fact, our two-speed relaxation solver with the choice (3.11) of $a$ and $b$ increases (respectively decreases) the numerical diffusion on the mass (resp. momentum) equations. This transfer of numerical diffusion between the mass and momentum equations was present in [24] and is crucial here for the validity of the entropy inequality in the low Mach regime. It is related to the idea of preconditioning [44].

\subsection{Asymptotic properties of the low Mach scheme}

In this subsection we prove the asymptotic-preserving property $a$ ) stated in Theorem 2. We then prove the statement $b$ ), and finally discuss the behaviour of the CFL condition (2.37) and the existence of checkerboard modes.

Proof of property a) of Theorem 2. Let us assume that the solution is in the low Mach regime. Then $\nabla_{x} p=\mathcal{O}(1)$, which implies that at each interface

$$
\pi^{R}-\pi^{L}=p^{R}-p^{L}=\mathcal{O}(1),
$$

or equivalently (taking into account (3.2)

$$
\rho^{R}-\rho^{L}=\mathcal{O}\left(M^{2}\right) .
$$


The consistency error can be evaluated by expanding the numerical flux minus the centered flux, i.e. the difference $F\left(U^{L}, U^{R}\right)-\left(F\left(U^{L}\right)+F\left(U^{R}\right)\right) / 2$. The numerical flux is defined by (2.35). According to the values of $\sigma^{L}, \sigma^{R}$ in (2.32) and the fact that $a^{L}, a^{R} \rightarrow \infty$ as $M \rightarrow 0$, one has $\sigma^{L} \rightarrow-\infty$, $\sigma^{R} \rightarrow \infty$. It implies that the value of the numerical flux in $(2.35)$ is $F^{L \star}$ or $F^{R \star}$, that are defined by $(2.36)$ in terms of the intermediate values. Let us rewrite the intermediate states (2.31) in terms of $M$, by using the definitions (3.2), (3.11), 2.71), 2.72). Easy expansions show that when the Mach number goes to zero, we have

$$
X^{\alpha}=\mathcal{O}(M), \quad b^{\alpha}=\bar{b}^{\alpha}+\mathcal{O}(M), \quad a^{\alpha}=\frac{\bar{b}^{\alpha}}{M^{2}}(1+\mathcal{O}(M)),
$$

with

$$
\frac{\bar{b}^{\alpha}}{\rho^{\alpha}}=\sqrt{\bar{p}^{\prime \alpha}}
$$

Moreover according to 3.15, (3.17) one has

$$
\bar{b}^{R}-\bar{b}^{L}=\mathcal{O}\left(M^{2}\right),
$$

thus we can write just $\bar{b}$ instead of $\bar{b}^{R}$ or $\bar{b}^{L}$ (up to $\mathcal{O}\left(M^{2}\right)$ errors). The intermediate states 2.31) can then be expanded as

$$
\begin{aligned}
\pi^{\star} & =\frac{\pi^{L}+\pi^{R}}{2}+\bar{b} \frac{u^{L}-u^{R}}{2}+\mathcal{O}\left(M\left(\pi^{L}-\pi^{R}\right)\right)+\mathcal{O}\left(M\left(u^{L}-u^{R}\right)\right) \\
v^{\star} & =\frac{u^{L}+u^{R}}{2}+\frac{\pi^{L}-\pi^{R}}{2 \bar{b}}+\mathcal{O}\left(M\left(\pi^{L}-\pi^{R}\right)\right)+\mathcal{O}\left(M\left(u^{L}-u^{R}\right)\right) \\
\frac{1}{\rho^{L \star}} & =\frac{1}{\rho^{L}}+\mathcal{O}\left(M^{2}\left(\pi^{L}-\pi^{R}\right)\right)+\mathcal{O}\left(M^{2}\left(u^{L}-u^{R}\right)\right) \\
\frac{1}{\rho^{R \star}} & =\frac{1}{\rho^{R}}+\mathcal{O}\left(M^{2}\left(\pi^{L}-\pi^{R}\right)\right)+\mathcal{O}\left(M^{2}\left(u^{L}-u^{R}\right)\right) \\
u^{L \star} & =u^{L}+\mathcal{O}\left(M^{2}\left(\pi^{L}-\pi^{R}\right)\right)+\mathcal{O}\left(M^{2}\left(u^{L}-u^{R}\right)\right) \\
u^{R \star} & =u^{R}+\mathcal{O}\left(M^{2}\left(\pi^{L}-\pi^{R}\right)\right)+\mathcal{O}\left(M^{2}\left(u^{L}-u^{R}\right)\right)
\end{aligned}
$$

We observe then that because of (3.2) and since $\bar{p}^{\prime}$ is bounded and non-zero, we have $\rho^{L}-\rho^{R}=\mathcal{O}\left(M^{2}\left(\pi^{L}-\pi^{R}\right)\right)$. Therefore $\rho^{L \star}, \rho^{R \star}$ are both close to $\rho^{L}\left(\right.$ or $\left.\rho^{R}\right)$ up to an error $\mathcal{O}\left(M^{2}\left(\pi^{L}-\pi^{R}\right)\right)+\mathcal{O}\left(M^{2}\left(u^{L}-u^{R}\right)\right)$. With 2.36 
we thus get

$$
\begin{aligned}
& F^{L \star}-\frac{F\left(U^{L}\right)+F\left(U^{R}\right)}{2} \\
= & \rho^{L}\left(v^{\star}-\frac{u^{L}+u^{R}}{2}, u^{L \star} v^{\star}-\frac{\left(u^{L}\right)^{2}+\left(u^{R}\right)^{2}}{2}\right)+\left(0, \pi^{\star}-\frac{\pi^{L}+\pi^{R}}{2}\right) \\
& +\mathcal{O}\left(M^{2}\left(\pi^{L}-\pi^{R}\right)\right)+\mathcal{O}\left(M^{2}\left(u^{L}-u^{R}\right)\right) \\
= & \rho^{L}\left(\frac{\pi^{L}-\pi^{R}}{2 \bar{b}}, u^{L} \frac{\pi^{L}-\pi^{R}}{2 \bar{b}}\right)+\left(0,\left(\rho^{L} u^{R}+\bar{b}\right) \frac{u^{L}-u^{R}}{2}\right) \\
& +\mathcal{O}\left(M\left(\pi^{L}-\pi^{R}\right)\right)+\mathcal{O}\left(M\left(u^{L}-u^{R}\right)\right) .
\end{aligned}
$$

Similarly we have

$$
\begin{aligned}
& F^{R \star}-\frac{F\left(U^{L}\right)+F\left(U^{R}\right)}{2} \\
= & \rho^{R}\left(\frac{\pi^{L}-\pi^{R}}{2 \bar{b}}, u^{R} \frac{\pi^{L}-\pi^{R}}{2 \bar{b}}\right)+\left(0,\left(-\rho^{R} u^{L}+\bar{b}\right) \frac{u^{L}-u^{R}}{2}\right) \\
& +\mathcal{O}\left(M\left(\pi^{L}-\pi^{R}\right)\right)+\mathcal{O}\left(M\left(u^{L}-u^{R}\right)\right) .
\end{aligned}
$$

Now since we are close to a smooth solution satisfying $(3.9)$, we have that $\left(\rho-\rho^{0}\right) / M^{2}$ is smooth and bounded (in the sense that $\nabla_{x}\left(\rho-\rho^{0}\right) / M^{2}$ is bounded also). We can thus write with $(3.2)$

$$
\pi^{L}-\pi^{R}=\frac{\bar{p}\left(\rho^{L}\right)-\bar{p}\left(\rho^{R}\right)}{M^{2}}=-\Delta x \bar{p}^{\prime} \partial_{x}\left(\frac{\rho-\rho^{0}}{M^{2}}\right)+\mathcal{O}\left(\Delta x^{2}\right),
$$

and $u^{L}-u^{R}=-\Delta x \partial_{x} u+\mathcal{O}\left(\Delta x^{2}\right)$. Indeed this is valid in $1 \mathrm{~d}$, but in multi-d a similar expansion is possible in terms of the size of the cell. The previous expansions of $F^{L \star}, F^{R \star}$ and the value $\bar{b}=\rho \sqrt{\bar{p}^{\prime}}$ lead to (3.12). The term $|u|$ indeed comes from the fact that the value of the numerical flux is either $F^{L \star}$ if $\sigma^{\star} \geq 0$ or $F^{R \star}$ if $\sigma^{\star} \leq 0$, noticing that $\sigma^{\star}=u+\mathcal{O}(\Delta x)$.

The expansion (3.12) means that the scheme is first-order uniformly in $M$. Moreover, excluding the factor $\Delta x$, the numerical viscosity in $u$ is bounded independently of $M$, and the numerical viscosity in $\rho$ is in $1 / M^{2}$. This large value is indeed affordable in the low Mach regime because of (3.15). Thus $a$ ) of Theorem 2 is proved, as well as (3.12).

Proof of property b) of Theorem 2. We have to prove that for $M<\sqrt{k \Delta x}$ (with $k$ a constant), the scheme is consistent at first order with the incompressible limit model (3.6), (3.7). In other words we have to prove that for $M$ and $\Delta x$ satisfying the previous inequality, the approximate solution $u_{M, \Delta x}$ is close to the exact solution $u$ to the incompressible system (3.6), (3.7), up to an error of order $\Delta x$. Consider the exact solution $\left(\rho_{M}, u_{M}\right)$ to the compressible system (3.1). According to property a) of Theorem 2 one has that $u_{M, \Delta x}-u_{M}$ is of order $\Delta x$. But since $u_{M}-u$ is of order $M^{2}$ according to 3.10 ) and $M^{2}=\mathcal{O}(\Delta x)$, we conclude that $u_{M, \Delta x}-u=\mathcal{O}(\Delta x)$, 
which is the claim. To finish the proof of $b$ ) of Theorem 2 , it remains to prove the uniformity with respect to time. In order to do that we use the relative entropy. Recall that the entropy $\eta(U)$ is defined by $(2.5)$. We define the entropy relative to the constant state $U^{\text {cst }}=\left(\rho^{0}, 0\right)$ by

$$
\begin{aligned}
\eta^{r e l}(U) & =\eta(U)-\eta\left(U^{c s t}\right)-\eta^{\prime}\left(U^{c s t}\right)\left(U-U^{c s t}\right) \\
& =\rho \frac{u^{2}}{2}+\rho e(\rho)-\rho^{0} e\left(\rho^{0}\right)-\left(e\left(\rho^{0}\right)+p\left(\rho^{0}\right) / \rho^{0}\right)\left(\rho-\rho^{0}\right) \\
& =\rho \frac{u^{2}}{2}+\frac{1}{M^{2}}\left(\rho \bar{e}(\rho)-\rho^{0} \bar{e}\left(\rho^{0}\right)-\left(\bar{e}\left(\rho^{0}\right)+\bar{p}\left(\rho^{0}\right) / \rho^{0}\right)\left(\rho-\rho^{0}\right)\right) .
\end{aligned}
$$

Since $\eta$ is convex, $\eta^{r e l}$ is nonnegative. Since our scheme satisfies a discrete entropy inequality for $\eta$, it satisfies also a discrete entropy inequality for $\eta^{r e l}$. Discretely integrating it in space (under suitable boundary conditions) and in time we get that

$$
\sum_{i}\left|C_{i}\right| \eta^{r e l}\left(U_{i}^{n}\right) \leq \sum_{i}\left|C_{i}\right| \eta^{r e l}\left(U_{i}^{0}\right),
$$

where $\left|C_{i}\right|$ is the volume of the cell $C_{i}$ associated with the finite volume method, and $U_{i}^{0}$ is the initial data. Using that for $\rho$ close to $\rho^{0}, \eta^{r e l}(U) \simeq$ $\rho u^{2} / 2+\bar{p}^{\prime}\left(\rho^{0}\right)\left(\rho-\rho^{0}\right)^{2} / 2 \rho^{0} M^{2}$, we deduce that $\rho-\rho^{0}=\mathcal{O}(M)$ (in the $L^{2}$ sense) and that the integral of $\rho u^{2}$ is bounded, both uniformly in time, because this is true initially by assumption. Remark that this holds true for all weak solutions, whereas the stronger approximation $(3.9)$ is valid only for smooth solutions. With the discrete form the the mass conservation we conclude that $\operatorname{div} u=\mathcal{O}(M)+\mathcal{O}(\Delta x)+\mathcal{O}(\Delta t)$ in the weak discrete sense, uniformly in time. This proves that the scheme is consistent with the incompressibility equation (3.6) uniformly in time, and finishes the proof of b) of Theorem 2 .

Behaviour of the CFL condition. Let us examine the CFL condition 2.37) with the choice of the speeds (3.11). With the value 2.32 of $\sigma^{L}$ and $\sigma^{R}$ ( $\sigma^{\star}$ is in between, and thus it can be ignored) i.e. $\sigma^{L}=u^{L}-a^{L} / \rho^{L}$, $\sigma^{R}=u^{R}+a^{R} / \rho^{R}$, in the low Mach regime the CFL condition is mainly driven by $a^{L} / \rho^{L}$ and $a^{R} / \rho^{R}$. According to (3.11) these numbers behave as $a / \rho \sim \sqrt{\bar{p}^{\prime}} / M^{2}$. We thus get the order of magnitude

$$
\Delta t / \Delta x \sim M^{2} / \sqrt{\bar{p}^{\prime}},
$$

which is very restrictive in the low Mach number regime (but implicit versions can be developed).

Let us show however that a parabolic-type CFL condition can be obtained. We proceed following an idea introduced in [7]. First of all, we recall that when solving the compressible barotropic Euler equations with small Mach number $M$, we are close to an incompressible flow within a distance 
of the order of $M^{2}$, see (3.10). On the other hand, we aim at considering a first-order scheme, for which the consistency error is expected to be of the order of $\Delta x$. As a consequence, when $M^{2}<k \Delta x$ (with $k$ a normalizing constant), the compressible system and the incompressible limit differ no more than by an error of $\Delta x$. Both are therefore also close to the compressible system for which $M=\sqrt{k \Delta x}$ since the solution to the compressible system with this value of the Mach number is also close to the incompressible limit within a distance of the order of $\Delta x$. We conclude that in general we can

$$
\text { replace in the scheme } M \text { by } M_{\text {used }}=\max (M, \sqrt{k \Delta x}),
$$

without making an error of more than $\Delta x$, which means that we maintain the first-order accuracy of the scheme. Numerically we end up by replacing the parameter $M$ (everywhere in the numerical scheme) by $\max (M, \sqrt{k \Delta x})$, while keeping unchanged the normalized pressure $\bar{p}$. In this way the stiffness can be reduced to its minimal. With this modification, the CFL condition now behaves as

$$
\Delta t \sim \frac{\Delta x \max \left(M^{2}, k \Delta x\right)}{\sqrt{\bar{p}^{\prime}}} .
$$

When $M<\sqrt{k \Delta x}$ it is now a parabolic-type CFL condition. On the contrary in the case $M>\sqrt{k \Delta x}$, the case of a Mach number of order unity or of a fine mesh, this CFL condition (3.27) is a classical hyperbolic-type CFL condition.

However, we have to mention that doing this trick of replacing $M$ by $\max (M, \sqrt{k \Delta x})$ in the scheme, which is based on an error analysis for smooth solutions, can give quite poor results for discontinuous solutions, see subsection 3.5. A related delicate issue is the choice of the constant $k$, which is homogeneous to the inverse of a length. Nevertheless the trick works very well for computing incompressible flows, as done in [7]. Indeed this is the basis of the so-called artificial compressibility schemes, see for example [38], and this proves that for $M<\sqrt{k \Delta x}$ the scheme (with or without the replacement (3.26) ) is consistent at first order with the incompressible limit model.

Checkerboard modes. It is well-known that most of the asymptotic-preserving schemes exhibit nonphysical steady checkerboard modes in low Mach regimes, see for instance [23], [40], [25]. In our case, such nonphysical steady modes do not exist since we proved in section (2.6.2) that thanks to the validity of the entropy inequality, steady solutions are necessarily constant in space. This result holds true uniformly with respect to the Mach number. In other words, the validity of a discrete entropy inequality can be understood as a strong stability result which is enough to prevent the development of nonphysical checkerboard modes. Note that the appearence of checkerboard modes in some asymptotic-preserving schemes maybe related to the fact 
that these schemes reduce the numerical viscosity in the velocity, but do not strengthen the viscosity on the density.

\subsection{Numerical test cases}

In this section, the asymptotic-preserving property of the scheme is investigated numerically. We compare the results given by a Rusanov scheme, a standard (one-speed) relaxation scheme i.e. the choice (2.80) of the speeds, and our (two-speed) asymptotic-preserving scheme with the choice (3.11) of the speeds. We consider four test cases, that are all in the fully developed low Mach regime, contrarily to the test cases considered in [22, 27] that are transient tests corresponding to small final time of the order of $M$. The tests are two-dimensional and use cartesian meshes.

\subsubsection{Travelling vortex}

The first test case consists in the propagation of a vortex. It is taken from [34]. For this test case, an exact solution is available which makes it particularly useful in order to check the accuracy of the scheme in the low Mach number regime. The pressure law is $\bar{p}(\rho)=\rho^{2} / 2$, and the initial conditions are

$$
\left\{\begin{array}{l}
\rho(t=0, x, y)=110+M^{2}\left(\frac{1.5}{4 \pi}\right)^{2} \delta(r)(k(r)-k(\pi)), \\
u_{1}(t=0, x, y)=0.6+1.5(1+\cos (r)) \delta(r)(0.5-y), \\
u_{2}(t=0, x, y)=0+1.5(1+\cos (r)) \delta(r)(x-0.5),
\end{array}\right.
$$

with

$$
k(r)=2 \cos (r)+2 r \sin (r)+\frac{\cos (2 r)}{8}+\frac{r \sin (2 r)}{4}+\frac{3 r^{2}}{4},
$$

and

$$
r=4 \pi \sqrt{(x-0.5)^{2}+(y-0.5)^{2}}, \quad \delta(r)= \begin{cases}1 & r<\pi, \\ 0 & \text { otherwise }\end{cases}
$$

The initial density profile is displayed on Figure 4. The exact solution writes

$$
\begin{gathered}
\rho(t, x, y)=\rho(0, x-0.6 t, y), \\
u_{1}(t, x, y)=u_{1}(0, x-0.6 t, y), \quad u_{2}(t, x, y)=u_{2}(0, x-0.6 t, y),
\end{gathered}
$$

it satisfies $\operatorname{div} u=0$ for all times. The spatial domain is $[0,1] \times[0,1]$ and periodic boundary conditions are considered. On Figures 5, 6, 7, the density profiles are displayed at time $t=0.1$ for the Rusanov scheme (left), the relaxation scheme (middle) and the AP scheme (right) in the case $M=1$ for different meshes. On Figures 8, 9, 10, the density and velocity magnitude errors are displayed in terms of $M$ considering several mesh refinements. It is observed that as the Mach number goes to zero the velocity magnitude errors 
computed by the Rusanov and the one-speed relaxation schemes increase incorrectly while the error obtained with the two-speed relaxation scheme remains constant (and correctly decays as the mesh is refined). On Figures $13,14,15,16$, the density and velocity magnitude errors are displayed in terms of $\Delta x$ considering several Mach numbers. It is observed that for $M=1$ the three schemes behave correctly. However, considering a fixed small Mach number, it is observed that the Rusanov and the relaxation scheme do not converge (or converge very slowly for even smaller mesh sizes not reported here) when refining the mesh. On the contrary the AP scheme correctly converges when refining the mesh and a first-order convergence is obtained even at small Mach numbers. This is one of the main properties we are looking for. On Figure 11, the temporal evolution of the $L_{2}$ norm of the velocity is shown and on Figure 12 the temporal evolution of the relative $L_{1}$ error on the velocity divergence is displayed. This quantity is defined by

$$
\text { RelErrDiv }{ }^{n}=\frac{\operatorname{ErrDiv}^{n}}{\operatorname{Grad}^{n}},
$$

with

$$
\begin{gathered}
\operatorname{ErrDiv}^{n}=\Delta x \Delta y \sum_{i j}\left|\frac{\left(\rho u_{1}\right)_{i+1 j}^{n}-\left(\rho u_{1}\right)_{i-1 j}^{n}}{2 \Delta x}+\frac{\left(\rho u_{2}\right)_{i j+1}^{n}-\left(\rho u_{2}\right)_{i j-1}^{n}}{2 \Delta y}\right|, \\
\operatorname{Grad}^{n}=\Delta x \Delta y \sum_{i j}\left(\left(\frac{\left(\rho u_{1}\right)_{i+1 j}^{n}-\left(\rho u_{1}\right)_{i-1 j}^{n}}{2 \Delta x}\right)^{2}+\left(\frac{\left(\rho u_{2}\right)_{i+1 j}^{n}-\left(\rho u_{2}\right)_{i-1 j}^{n}}{2 \Delta x}\right)^{2}\right. \\
\left.+\left(\frac{\left(\rho u_{1}\right)_{i j+1}^{n}-\left(\rho u_{1}\right)_{i j-1}^{n}}{2 \Delta y}\right)^{2}+\left(\frac{\left(\rho u_{2}\right)_{i j+1}^{n}-\left(\rho u_{2}\right)_{i j-1}^{n}}{2 \Delta y}\right)^{2}\right)^{\frac{1}{2}} .
\end{gathered}
$$

It is observed that the relaxation scheme strongly damps the solution at small Mach number, whereas it is not the case with our AP scheme.

\subsubsection{Taylor-Green vortex}

Our second test is the well-known Taylor-Green vortex [7]. The initial conditions are displayed on Figure 17 and given by

$$
\begin{aligned}
& \rho(t=0, x, y)=1, \\
& u_{1}(t=0, x, y)=-\cos \left(w_{1} x\right) \sin \left(w_{2} y\right), \\
& u_{2}(t=0, x, y)=\frac{w_{1}}{w_{2}} \sin \left(w_{1} x\right) \cos \left(w_{2} y\right),
\end{aligned}
$$

with $w_{1}=3$ and $w_{2}=2$. The spatial domain is $[-\pi, \pi] \times[-\pi, \pi]$ and periodic boundary conditions are considered. In the incompressible limit, this is an exact steady solution. The pressure law is taken $\bar{p}(\rho)=\rho^{2} / 2$. On Figures 18, 19, the density and velocity magnitude errors are displayed 
with respect to the Mach number $M$ and for several mesh refinements. As in the previous test case, it is observed that when the Mach number goes to zero the velocity magnitude errors computed for the relaxation scheme increase incorrectly while the ones obtained with the AP scheme remain constant, and decays when refining the mesh. The temporal evolutions of the $L_{2}$ norm of the velocity and of the relative $L_{1}$ errors of the velocity divergence are displayed on Figures 20, 21, 22, Again, we observe that the relaxation scheme strongly damps the solution when the Mach number is small, whereas it is not the case with the AP scheme.

\subsubsection{Backward facing step flow}

In this test case, a viscosity term is added. The barotropic dimensionless Navier-Stokes equations under consideration then read

$$
\partial_{t}\left(\begin{array}{c}
\rho \\
\rho u
\end{array}\right)+\operatorname{div}_{x}\left(\begin{array}{c}
\rho u \\
\rho u \otimes u+\frac{\bar{p}(\rho)}{M^{2}} I d
\end{array}\right)=\left(\begin{array}{c}
0 \\
\frac{1}{R e} \Delta u
\end{array}\right),
$$

where $R e$ is the so-called Reynolds number. A standard finite-difference scheme is used for the viscous term. Our test case is taken from [30] and the initial conditions are $\rho=1, u=0$. The space domain is given by $[0, L=$ $8] \times[-0.5,0.5]$ and no flow boundary conditions are set ie $u=0$ for the top and bottom walls and for the left step in $(x=0,-0.5<y<0)$. The inflow boundary conditions in $(x=0,0<y<0.5)$ is $\left(u_{1}, u_{2}\right)=(12 y(1-2 y), 0)$ and the outflow boundary condition in $(x=L,-0.5<y<0.5)$ is $\left(u_{1}, u_{2}\right)=$ $\left(-3 y^{2}+3 / 4,0\right)$. In addition, these boundary conditions are ramped up from time 0 to time 1 by the function $(1-\cos (\pi t)) / 2$ and Neumann boundary conditions are set for the density.

For this test case, we set $M=0.01, R e=100, \Delta x=1 / 16$ and $\Delta y=$ $1 / 32$. The contour plots of the velocity and the velocity stream functions are displayed on Figures 23, 24, 25 for the Rusanov scheme (top), the relaxation scheme (middle) and our asymptotic-preserving scheme (bottom) at time $t=$ 20. It is observed that the Rusanov scheme does not capure the recirculation unlike the relaxation and the AP schemes. However, the relaxation scheme does not obtain the correct reattachment point position and underestimates it while the AP scheme gives very close results to the ones obtained in [30]. Again, this clearly shows the gain of the method proposed in the present work compared to standard numerical schemes for low Mach regime applications.

\subsubsection{Lid cavity}

This test case is with viscosity, as the previous one. It concerns a cubic cavity full of fluid where all the walls but one are fixed (in the present case the top 
wall of the cavity). The moving wall drags the fluid and initiates a global circulation of the fluid. A central main recirculation must be observed with smaller recirculations at the bottom corners. The initial conditions are $\rho=$ $1, u=0$ and the space domain is $[0,1] \times[0,1]$. No flow boundary conditions are set i.e. $u=0$ at the walls. Neumann boundary conditions are considered for the density and the velocity boundary condition at the moving wall is $\left(u_{1}, u_{2}\right)=(1,0)$. For this test case, we set $M=0.2, R e=400, \Delta x=\Delta y=$ $1 / 200$. The contour plots and the velocity and velocity stream functions are displayed on Figures 26, 27, 28 for the Rusanov scheme (top), the relaxation scheme (middle) and the asymptotic-preserving scheme (bottom) at time $t=30$. It is observed that the Rusanov scheme does not capture correctly the recirculations unlike the relaxation and AP schemes. The relaxation scheme and the AP scheme give close results, which is expected since the Mach number considered here is not very small. This also shows the good behaviour of the AP scheme in intermediate regimes.

\subsection{Limit of the approach and perspectives: toward an im- plicit two-speed relaxation scheme}

We would like here to point out some limits of the current approach for transitory regimes, and perspectives to go beyond.

Let us consider a basic test case in one space dimension, taken from [27]. It consists in a Riemann problem giving rise to a double rarefaction in a low Mach number regime. We are interested in the transitory regime when the final time is so short (of the order of $M$ ) that the acoustic waves are still present in the domain. The spatial domain is $[0,1]$, Dirichlet boundary conditions are considered and we take $M^{2}=10^{-3}, \bar{p}(\rho)=\rho^{2}$. The initial conditions are

$$
\rho(0, x)=\left\{\begin{array}{ll}
1+M^{2} & \text { if } x \in(0,0.5), \\
1 & \text { if } x \in(0.5,1),
\end{array} \quad u(0, x)= \begin{cases}1-M & \text { if } x \in(0,0.5), \\
1+M & \text { if } x \in(0.5,1) .\end{cases}\right.
$$

On Figure 29 the density profile (left) and momentum profile (right) are displayed at initial time and at time $t=7 \times 10^{-3}$. The initial condition is in green and the exact solution is in black. A spacestep of $\Delta x=1 / 800$ is used. Figure 29 shows the results obtained with the AP scheme in the context of the replacement (3.26), which means that in the pressure law (3.2) and in the algorithm (but not in the above initial data!), $M$ is replaced by $M_{\text {used }}$, with $M_{\text {used }}=\max (M, \sqrt{k \Delta x})$. The choice $M_{\text {used }}=\sqrt{\Delta x}(k=1)$ is shown in blue and the choice $M_{\text {used }}=M$ is shown in red. It is observed that the modification $M_{\text {used }}=\sqrt{\Delta x}$ leads to inaccurate results in this transitory regime (especially on the intermediate states), although $\sqrt{\Delta x} \simeq 0.035$ is only slighly greater than $M \simeq 0.031$. On the contrary, the AP scheme with $M_{\text {used }}=M$ captures the correct solution, but the CFL condition involves the factor $M^{2}$ according to 3.25 . We recall that the modification 
$M_{\text {used }}=\max (M, \sqrt{k \Delta x})$ was introduced to avoid such a restrictive CFL condition and switch to the parabolic CFL condition (3.27).

Perspectives and work in progress. In order to avoid both the replacement (3.26) and the stringent CFL restriction (3.25) in low Mach number regimes, an implicit scheme can be considered. This can be done following for instance the ideas introduced in [12, 14, 15]. More precisely, a LagrangeProjection like decomposition is performed such that the Lagrange system contains the stiff acoustic part. This Lagrange part is solved implicitly, whereas the projection step is kept explicit. Using our two-speed relaxation system to approximate the solution to the Lagrange system will make the implicit resolution very cheap in terms of computational cost since it is associated with a quasilinear system. At last, this implicitation of the acoustic part would enable to avoid both the introduction of the parameter $M_{\text {used }}$ and the CFL condition, and lead to accurate results also in transitory regimes. A standard hyperbolic CFL condition associated with the explicit projection step would ensure the stability of the overall scheme. A rigorous study of this implicit-explicit approach in the framework of the AP scheme presented here is beyond the scope of this paper and is currently investigated.

\section{Summary and conclusion}

We have introduced a new relaxation model of Suliciu type for the barotropic Euler equations. Contrarily to the clasical one, our relaxation system has two speeds as parameters. Under subcharacteristic conditions on these speeds, the associated solver satisfies a discrete entropy inequality, and keeps the positivity of the density. The advantage of having two speeds is that we can monitor independently the numerical viscosity in the density and velocity variables. We have applied our solver to the barotropic Euler equations in the low Mach number regime. In this context it is well-known that standard schemes put too much numerical viscosity on the velocity (proportionally to $1 / M$, where $M$ is the Mach number). Schemes that are modified to handle correctly the low Mach regime are classically called asymptotic preserving, which means that they are uniformly accurate. However for all such schemes in the literature, the applied modifications prevent the scheme from satisfying a discrete entropy inequality. For our two-speed relaxation solver we have proved that some particular explicit choices of the relaxation speeds enable to establish for the first time both the asymptotic-preserving property and a discrete entropy inequality. This strong property is related to the possibility of monitoring correctly the numerical viscosity coefficients. Indeed one has to keep a finite numerical viscosity in the velocity variable, whereas the numerical viscosity in the density variable has to grow like $1 / M^{2}$. This large value is possible because the density is close to a constant in the low 
Mach regime. Recall that in a classical Godunov solver the viscosities on the density and velocity are of the same order $1 / M$. The discrete entropy inequality satisfied by our scheme is a strong stability property that allows us to prove that for small Mach number $M$ our scheme is consistent with the incompressible Euler equations, that the incompressibility condition is approximated uniformly in time, and that checkerboard modes do not exist.

Two-dimensional test cases have been presented to confirm our theoretical properties. A limit of our approach is that our scheme is explicit, and the CFL condition blows up like $1 / M^{2}$ in the low Mach regime. We have proposed a modification (3.26) of our scheme in order to avoir this blow up and to reduce to a parabolic-type CFL condition in the low Mach regime. However this modification gives inaccurate results in transitory regime when the time is of the order of the Mach number. Therefore, in order to avoid both the use of our modification and the restrictive CFL condition, an implicit two-speed relaxation model will be considered in a forthcoming work, using the approach of [14].

\section{References}

[1] F. Berthelin and F. Bouchut. Kinetic invariant domains and relaxation limit from a BGK model to isentropic gas dynamics. Asymptotic analysis, 31:153-176, 2002.

[2] C. Berthon, P. Charrier, and B. Dubroca. An HLLC scheme to solve the M1 model of radiative transfer in two space dimensions. J. Sci. Comput., 31:347-389, 2007.

[3] F. Bouchut. A reduced stability condition for nonlinear relaxation to conservation laws. J. Hyp. Diff. Eq., 1:149-170, 2004.

[4] F. Bouchut. Nonlinear stability of finite volume methods for hyperbolic conservation laws, and well-balanced schemes for sources. Frontiers in Mathematics. Birkhäuser Verlag, Basel, 2004.

[5] F. Bouchut and S. Boyaval. A new model for shallow viscoelastic fluids. Math. Models Meth. Appl. Sci., 23:1479-1526, 2013.

[6] F. Bouchut and H. Frid. Finite difference schemes with cross derivatives correctors for multidimensional parabolic systems. J. Hyp. Diff. Eq., $3: 27-52,2006$.

[7] F. Bouchut, Y. Jobic, R. Natalini, R. Occelli, and V. Pavan. Secondorder entropy satisfying BGK-FVS schemes for incompressible NavierStokes equations. SMAI J. Comput. Math., 4:1-56, 2018. 
[8] F. Bouchut, C. Klingenberg, and K. Waagan. A multiwave approximate Riemann solver for ideal MHD based on relaxation II - Numerical implementation with 3 and 5 waves. Numer. Math., 115:647-679, 2010.

[9] F. Bouchut and X. Lhébrard. A 5-wave relaxation solver for the shallow water MHD system. J. Scientif. Comput., 68:92-115, 2016.

[10] F. Bouchut and T. Morales. Semi-discrete entropy satisfying approximate Riemann solvers. The case of the Suliciu relaxation approximation. J. Scient. Comput., 41:483-509, 2009.

[11] C. Chalons and F. Coquel. Modified Suliciu relaxation system and exact resolution of isolated shock waves. Math. Models Methods Appl. Sci., 24:937-971, 2014.

[12] C. Chalons, F. Coquel, and C. Marmignon. Well-balanced time implicit formulation of relaxation schemes for the Euler equations. SIAM J. Sci. Comput., 30:394-415, 2007.

[13] C. Chalons and J.-F. Coulombel. Relaxation approximation of the Euler equations. J. Math. Anal. Applic., 348:872-893, 2008.

[14] C. Chalons, M. Girardin, and S. Kokh. An all-regime LagrangeProjection like scheme for the gas dynamics equations on unstructured meshes. Commu. Comput. Phys., 20:188-233, 2016.

[15] C. Chalons, M. Girardin, and S. Kokh. An all-regime LagrangeProjection like scheme for 2D homogeneous models for two-phase flows on unstructured meshes. J. Comput. Phys., 335:885-904, 2017.

[16] G.-Q. Chen, C.-D. Levermore, and T.-P. Liu. Hyperbolic conservation laws with stiff relaxation terms and entropy. Comm. Pure Appl. Math., 48:787-830, 1995.

[17] P. Colella and K. Pao. A Projection Method for Low Speed Flows. J. Comput. Physics, 149:245-269, 1999.

[18] F. Coquel, E. Godlewski, A. In, B. Perthame, and P. Rascle. Some new Godunov and relaxation methods for two phase flows. In Proceedings of an International conference on Godunov methods : Theory and Applications. Kluwer Academic/Plenum, 2001.

[19] F. Coquel, E. Godlewski, and N. Seguin. Relaxation of fluid systems. Math. Models Methods Appl. Sci., 22:1250014, 2012.

[20] F. Cordier, P. Degond, and A. Kumbaro. An Asymptotic-Preserving all-speed scheme for the Euler and Navier-Stokes equations. J. Comput. Phys., 231:5685-5704, 2012. 
[21] P. Degond, S. Jin, and J.-G. Liu. Mach number uniform asymptoticpreserving gauge schemes for compressible flows. Bull. Inst. Math., Acad. Sin., 2:851-892, 2007.

[22] P. Degond and M. Tang. All speed method for the Euler equation in the low mach number limit. Comm. Comput. Phys., 10:1-31, 2011.

[23] S. Dellacherie. Checkerboard modes and wave equation. In Proc. of the Algoritmy 2009 Conference on Scientic Computing (March 15-20, 2009, Vysoke Tatry, Podbanske, Slovakia). 2009.

[24] S. Dellacherie. Analysis of Godunov type schemes applied to the compressible euler system at low Mach number. J. Comput. Phys., 229:9781016, 2010.

[25] S. Dellacherie, J. Jung, P. Omnes, and P.-A. Raviart. Construction of modified Godunov type schemes accurate at any Mach number for the compressible Euler system. Math. Models Meth. Appl. Sci., 26:2525$2615,2016$.

[26] B. Després. Lagrangian systems of conservation laws. Invariance properties of Lagrangian systems of conservation laws, approximate Riemann solvers and the entropy condition. Numer. Math., 89:99-134, 2001.

[27] G. Dimarco, R. Loubere, and M.-H. Vignal. Study of a new Asymptotic Preserving schemes for the Euler system in the low Mach number limit. SIAM J. Scientific Comput., 39:A2099-A2128, 2017.

[28] J.-L. Guermond and B. Popov. Viscous regularization of the Euler equations and entropy principles. SIAM J. Appl. Math., 74:284-305, 2014.

[29] H. Guillard and C. Viozat. On the behavior of upwind schemes in the low Mach limit . Comp. \& Fluids, 28:63-86, 1999.

[30] J. Haack, S. Jin, and J.G. Liu. An all-speed asymptotic-preserving method for the isentropic Euler and Navier-Stokes equations. Comm. Computat. Phys., 12:955-980, 2012.

[31] A. Harten, P.D. Lax, and B. Van Leer. On upstream differencing and Godunov-type schemes for hyperbolic conservation laws. SIAM Review, 25:35-61, 1983.

[32] D. Iampietro, F. Daude, P. Galon, and J.-M. Hérard. A Mach-sensitive splitting approach for Euler-like systems. ESAIM: M2AN, 52:207-253, 2018 . 
[33] S. Jin and Z. Xin. The relaxation schemes for systems of conservation laws in arbitrary space dimension. Comm. Pure. Appl. Math., 48:235$276,1995$.

[34] K. Kaiser, J. Schutz, R. Schobel, and S. Noelle. A new stable splitting for the isentropic Euler equations. J. Scientif. Comput., 70:1390-1407, 2017.

[35] R. Klein. Semi-implicit extension of a Godunov-type scheme based on low Mach number asymptotics I: One-dimensional flow. J. Comput. Phys., 121:213-237, 1995.

[36] T.-P. Liu. Hyperbolic conservation laws with relaxation. Comm. Math. Phys., 108:153-175, 1987.

[37] R. Natalini. Convergence to equilibrium for the relaxation approximations of conservation laws. Comm. Pure Appl. Math., 49:1-30, 1996.

[38] T. Ohwada and P. Asinari. Artificial compressibility method revisited: Asymptotic numerical method for incompressible Navier-Stokes equations. J. Comput. Phys., 229:1698-1723, 2010.

[39] H. Paillere, C. Viozat, A. Kumbaro, and I. Toumi. Comparison of low mach number models for natural convection problems. Heat Mass Transfer, 36:567-573, 2000.

[40] R. Rieper. A low-Mach number fix for Roe's approximate Riemann solver. J. Computat. Phys., 230:5263-5287, 2011.

[41] A. Sangam. An HLLC scheme for ten-moments approximation coupled with magnetic field. Int. J. Comput. Sci. Math., 2:73-109, 2008.

[42] I. Suliciu. On the thermodynamics of fluids with relaxation and phase transitions. Fluids with relaxation. Int. J. Engrg. Sci., 36:921-947, 1998 .

[43] B. Thornber, A. Mosedale, D. Drikakis, D. Youngs, and R.J.R. Williams. An improved reconstruction method for compressible flows with low Mach number features. J. Comput. Phys., 227:4873-4894, 2008 .

[44] E. Turkel. Preconditioned methods for solving the incompressible and low speed compressible equations. J. Comput. Phys., 72:277-298, 1987. 


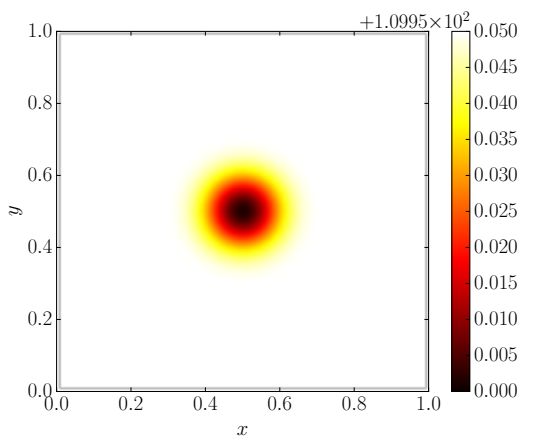

Figure 4: Initial density
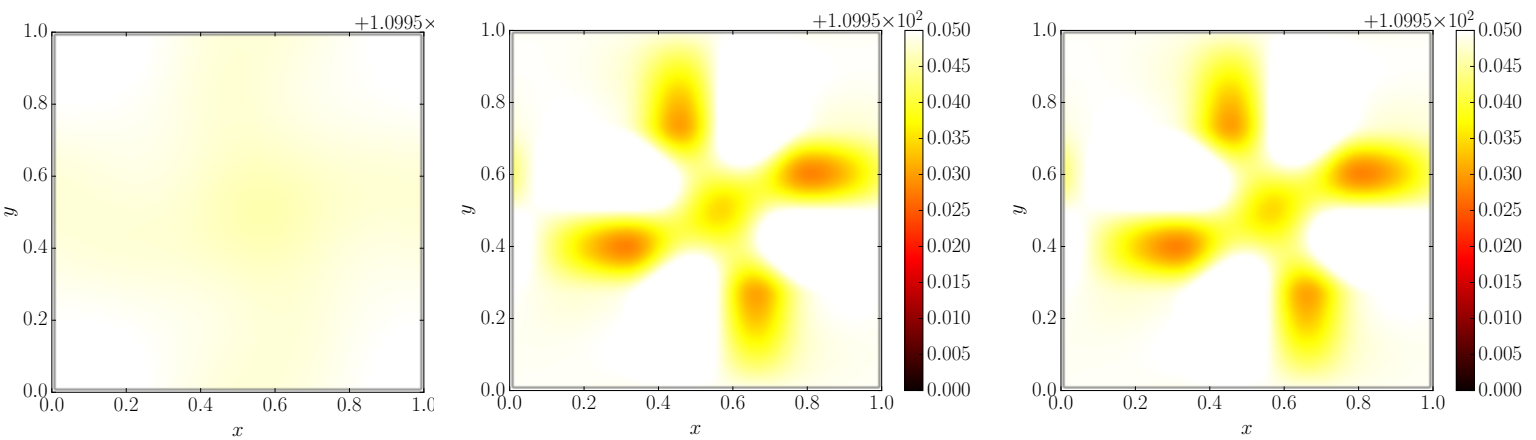

Figure 5: Density profile obtained with a Rusanov scheme (left), the relaxation scheme (middle) and the $\mathrm{AP}$ scheme (right) at $t=0.1$ for $M=1 \mathrm{in}$ the case $50 \times 50$.
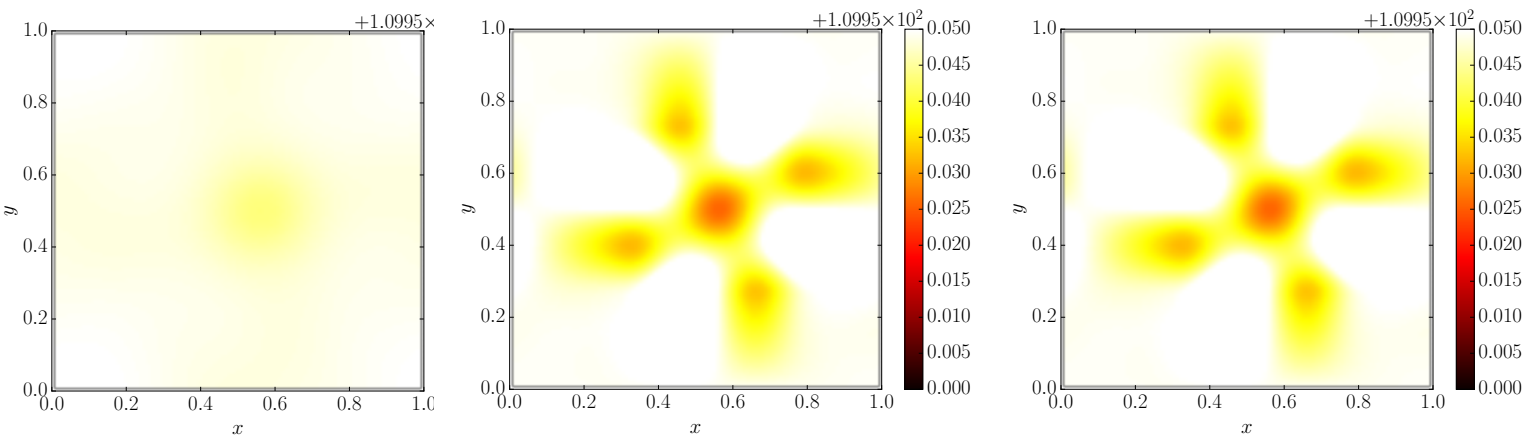

Figure 6: Density profile obtained with a Rusanov scheme (left), the relaxation scheme (middle) and AP scheme (right) at $t=0.1$ for $M=1$ in the case $100 \times 100$.
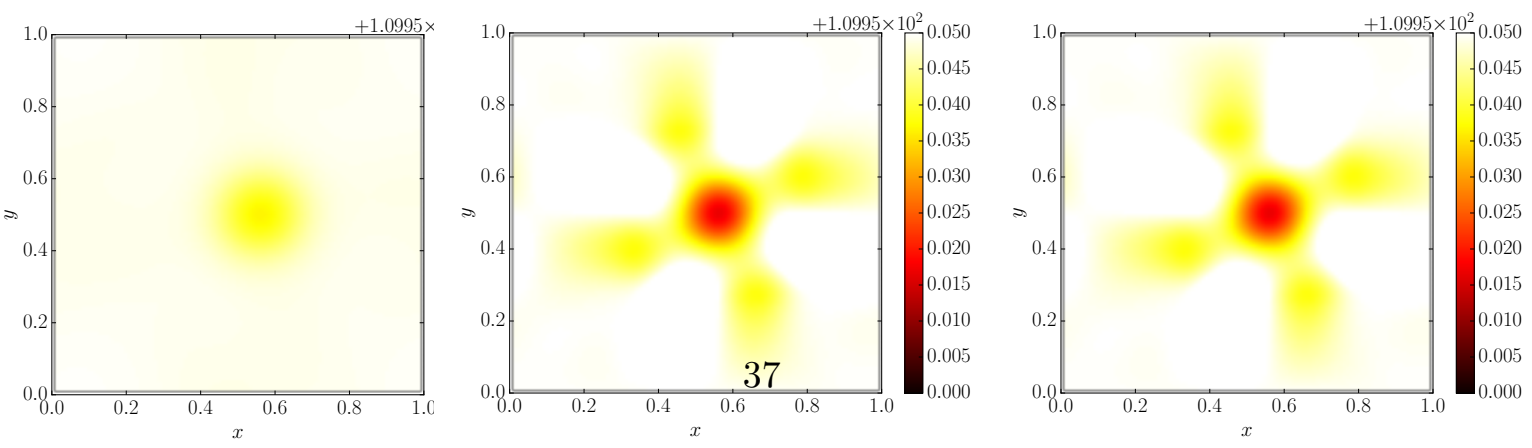

Figure 7: Density profile obtained with a Rusanov scheme (left), the relaxation scheme (middle) and AP scheme (right) at $t=0.1$ for $M=1$ in the case $200 \times 200$. 

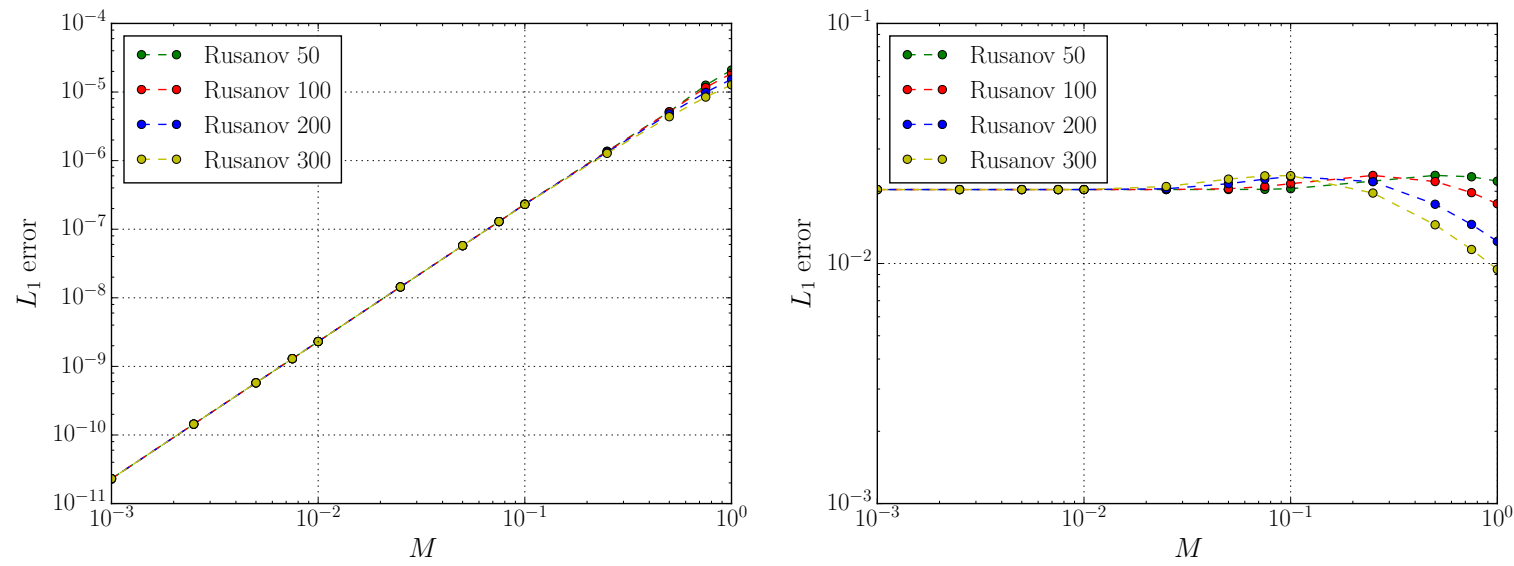

Figure 8: Density error (left) and velocity magnitude error (right) in terms of $M$ for the Rusanov scheme at time $t=0.1$ for different meshes (Travelling vortex).
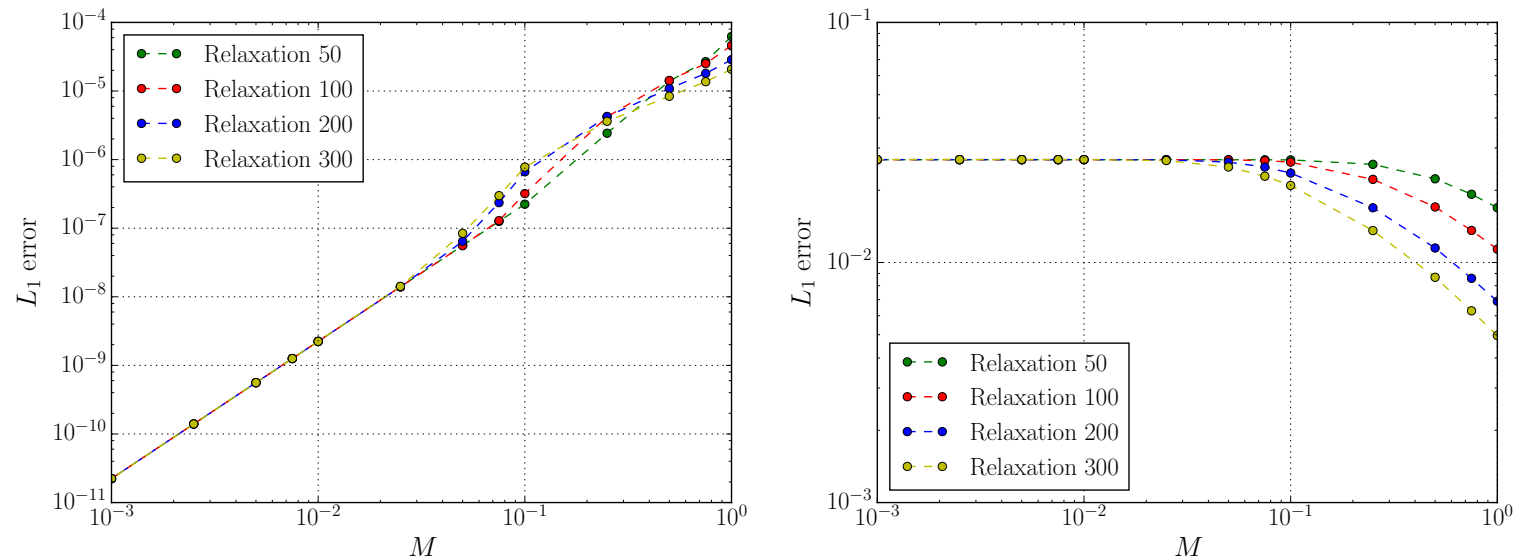

Figure 9: Density error (left) and velocity magnitude error (right) in terms of $M$ for the one-speed relaxation scheme at time $t=0.1$ for different meshes (Travelling vortex).
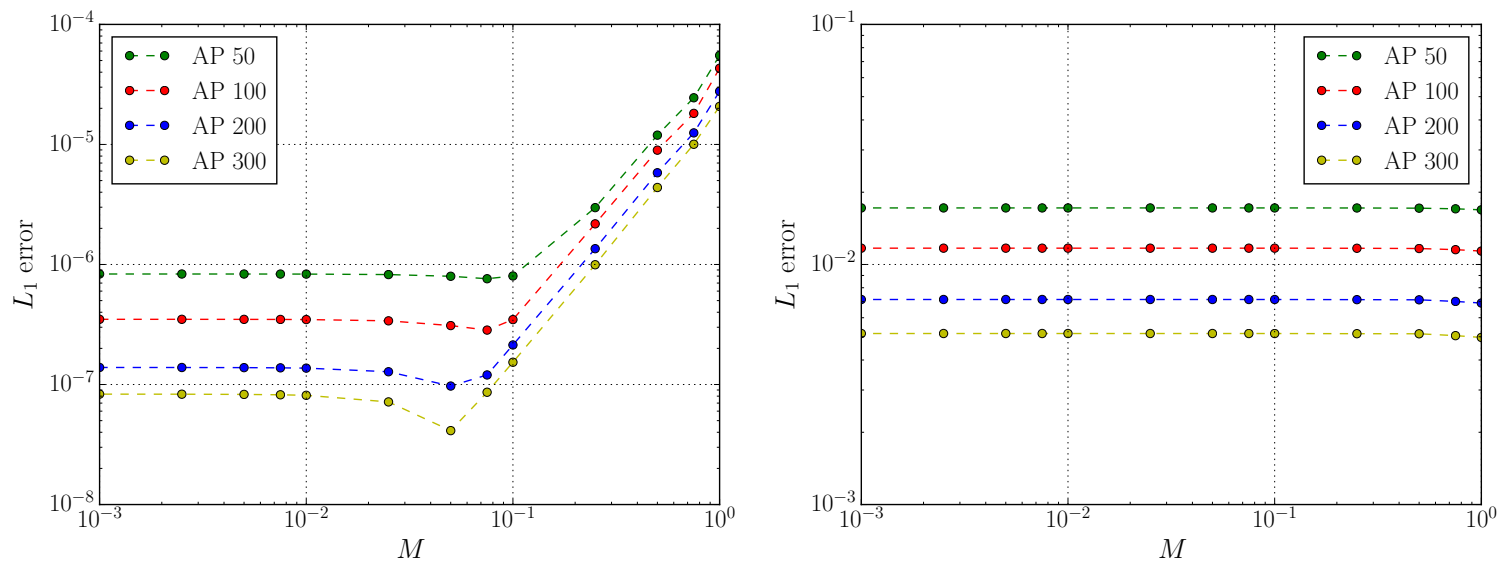

38

Figure 10: Density error (left) and velocity magnitude error (right) in terms of $M$ for the AP scheme at time $t=0.1$ for different meshes (Travelling vortex). 

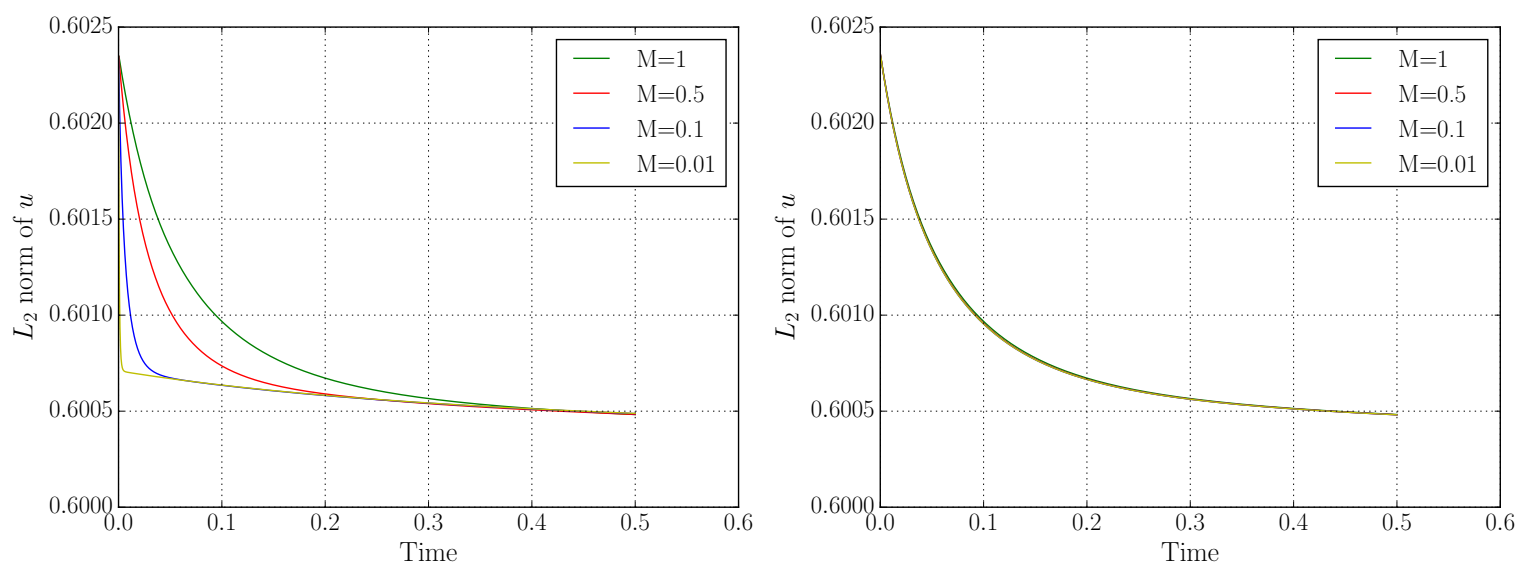

Figure 11: Temporal evolution of the $L_{2}$ norm of the velocity for the relaxation scheme (left) and the AP scheme (right) in the case $50 \times 50$ for different $M$ (Travelling vortex).
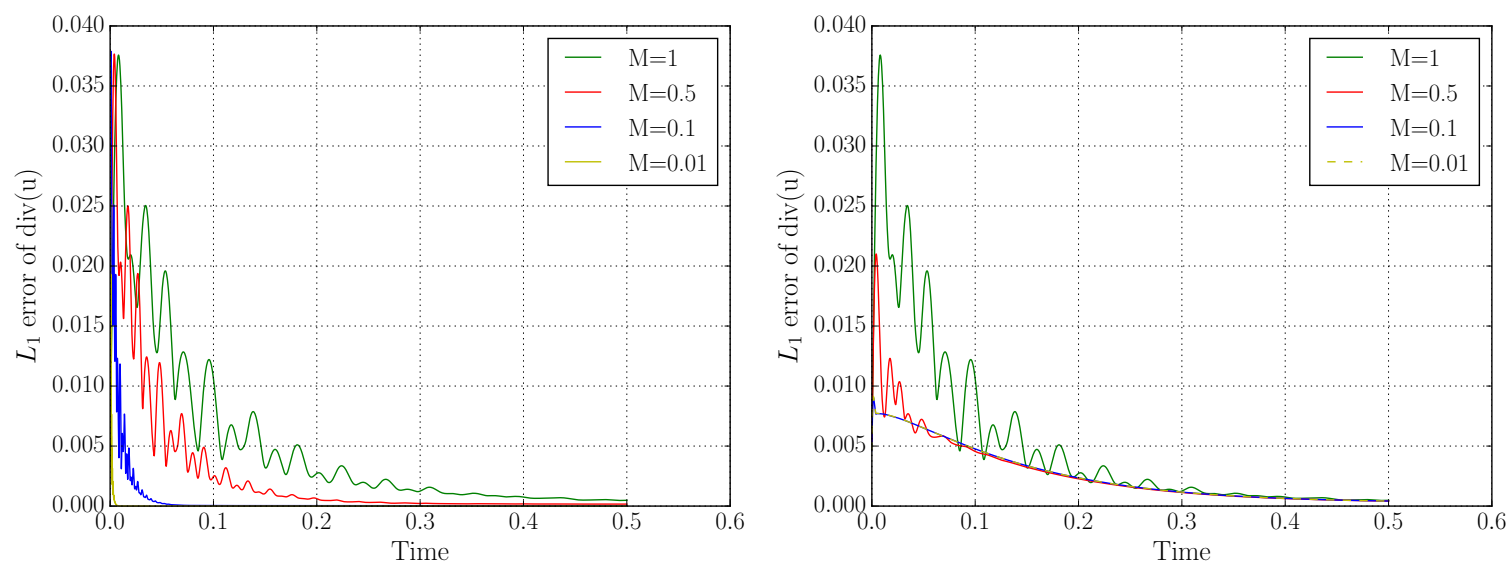

Figure 12: Temporal evolution of the relative $L_{1}$ error of the velocity divergence for the relaxation scheme (left) and the AP scheme (right) in the case $50 \times 50$ (Travelling vortex). 

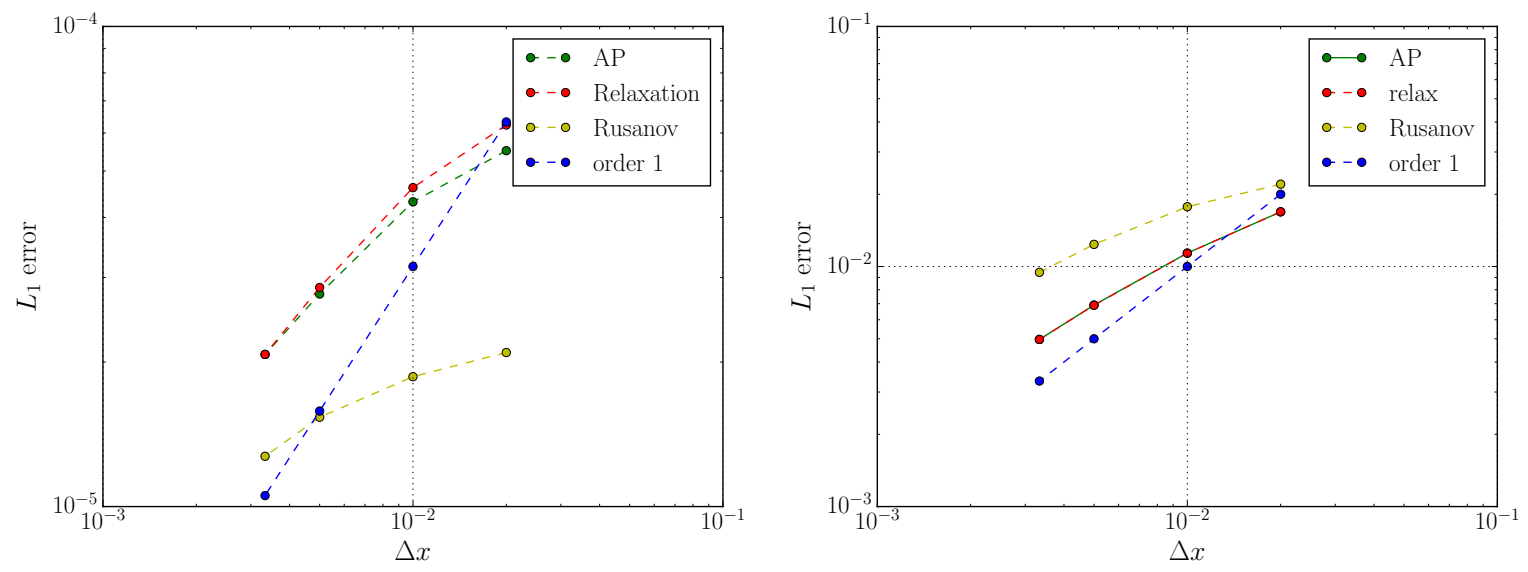

Figure 13: Density error (left) and velocity magnitude error (right) in terms of $\Delta x$ for $M=1$ (Travelling vortex).
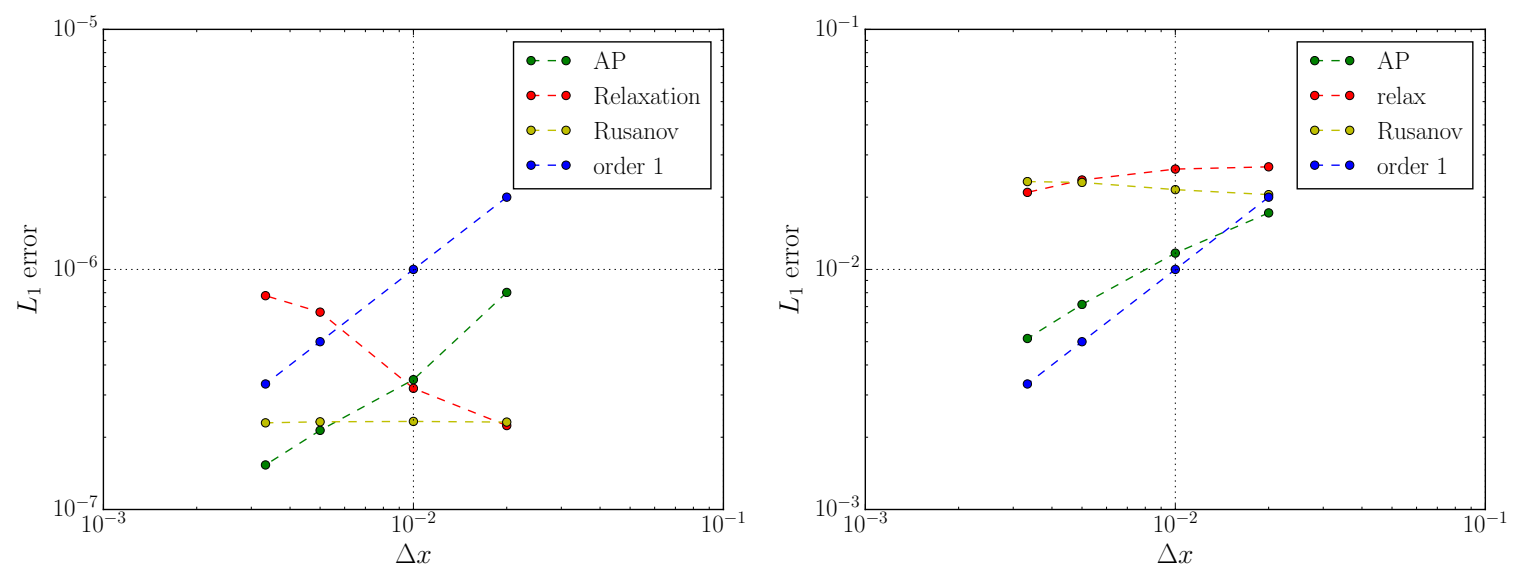

Figure 14: Density error (left) and velocity magnitude error (right) in terms of $\Delta x$ for $M=0.1$ (Travelling vortex).
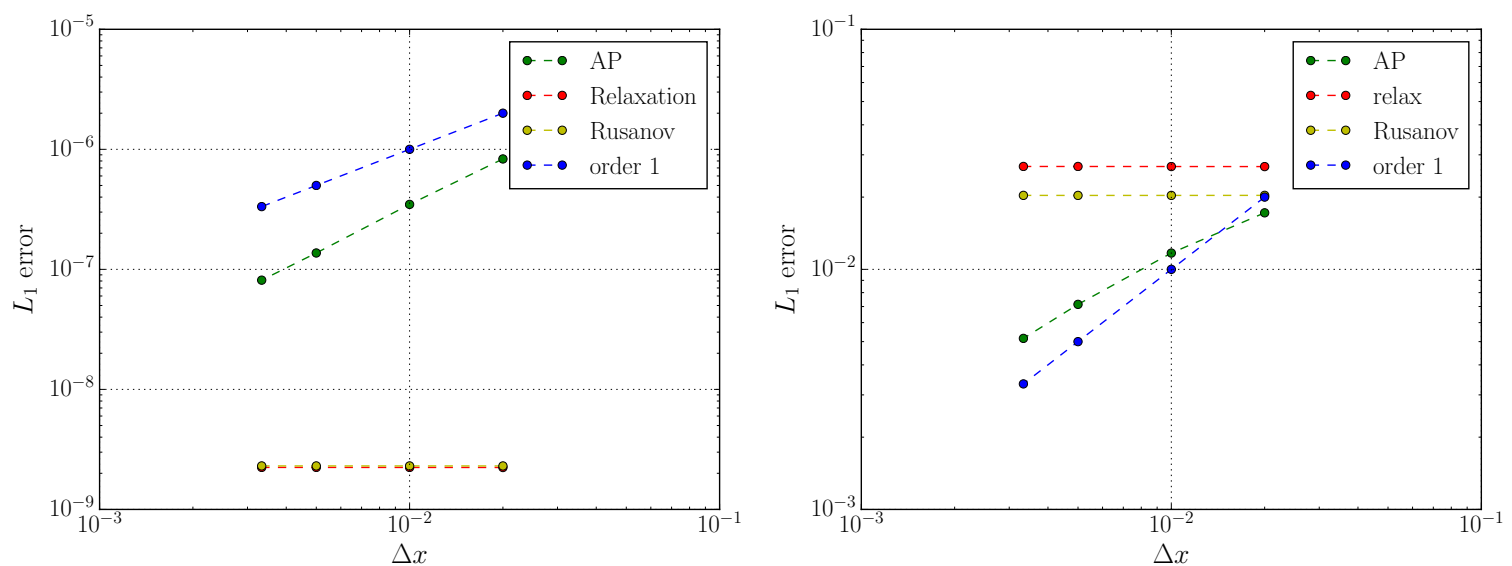

Figure 15: Density error (left) and velocity magnitude error (right) in terms of $\Delta x$ for $M=0.01$ (Travelling vortete). 

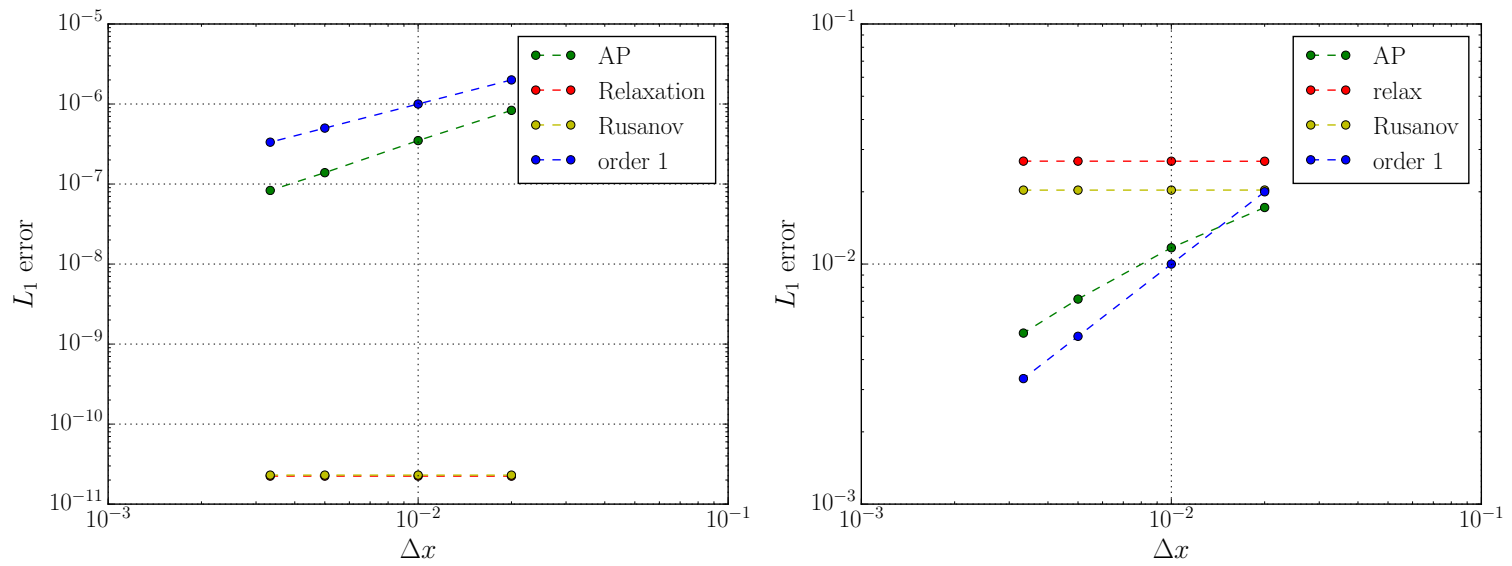

Figure 16: Density error (left) and velocity magnitude error (right) in terms of $\Delta x$ for $M=0.001$ (Travelling vortex).

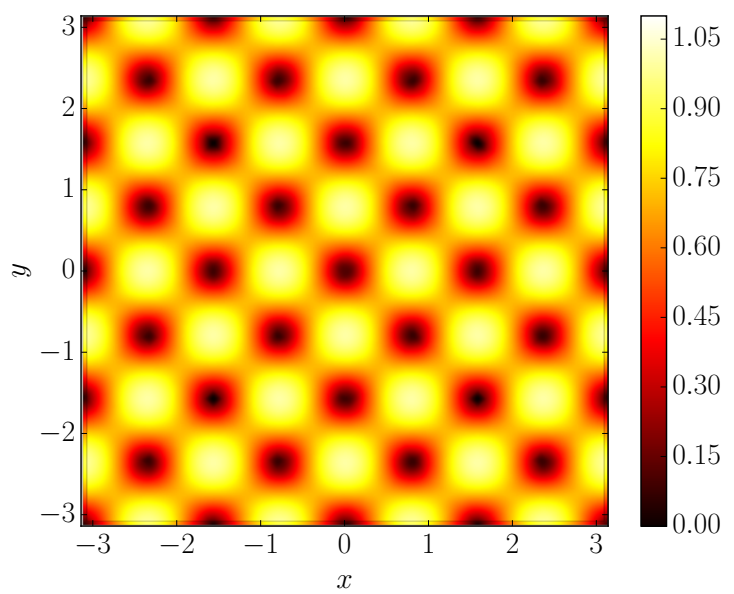

Figure 17: Initial velocity magnitude in the case $50 \times 50$. 

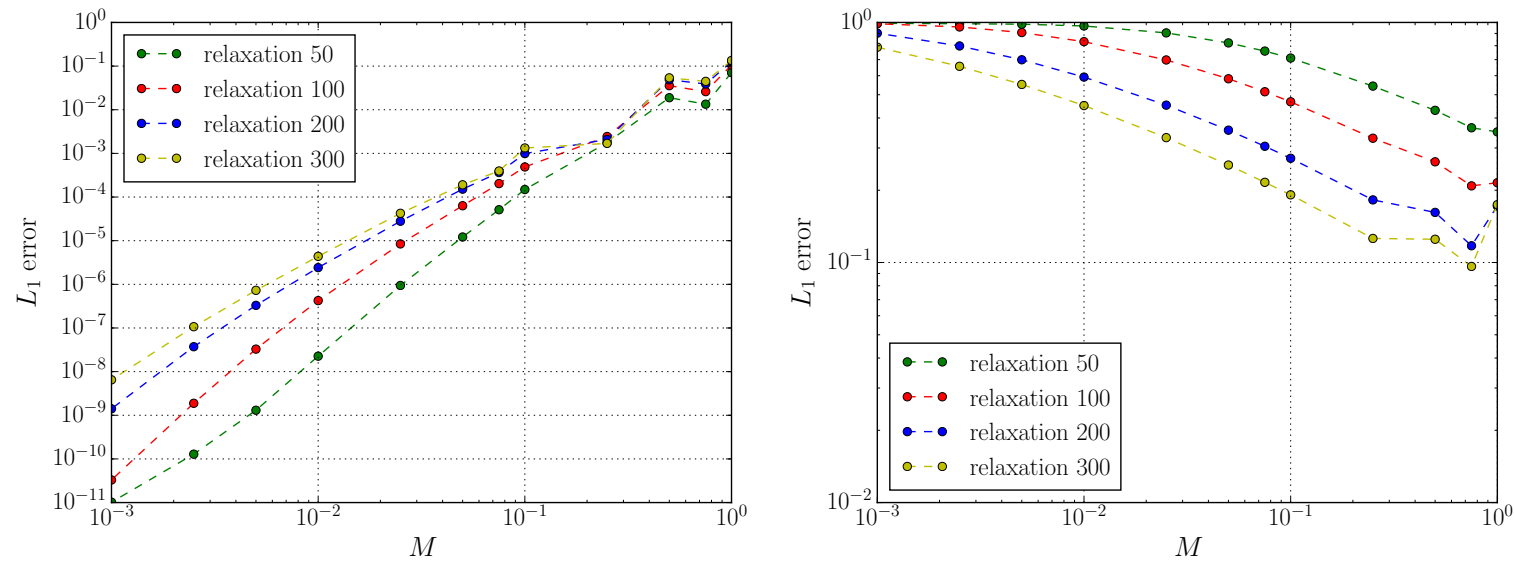

Figure 18: Density error (left) and velocity magnitude error (right) in terms of $M$ for the one-speed relaxation scheme at time $t=1$ for different meshes (Taylor-Green vortex).
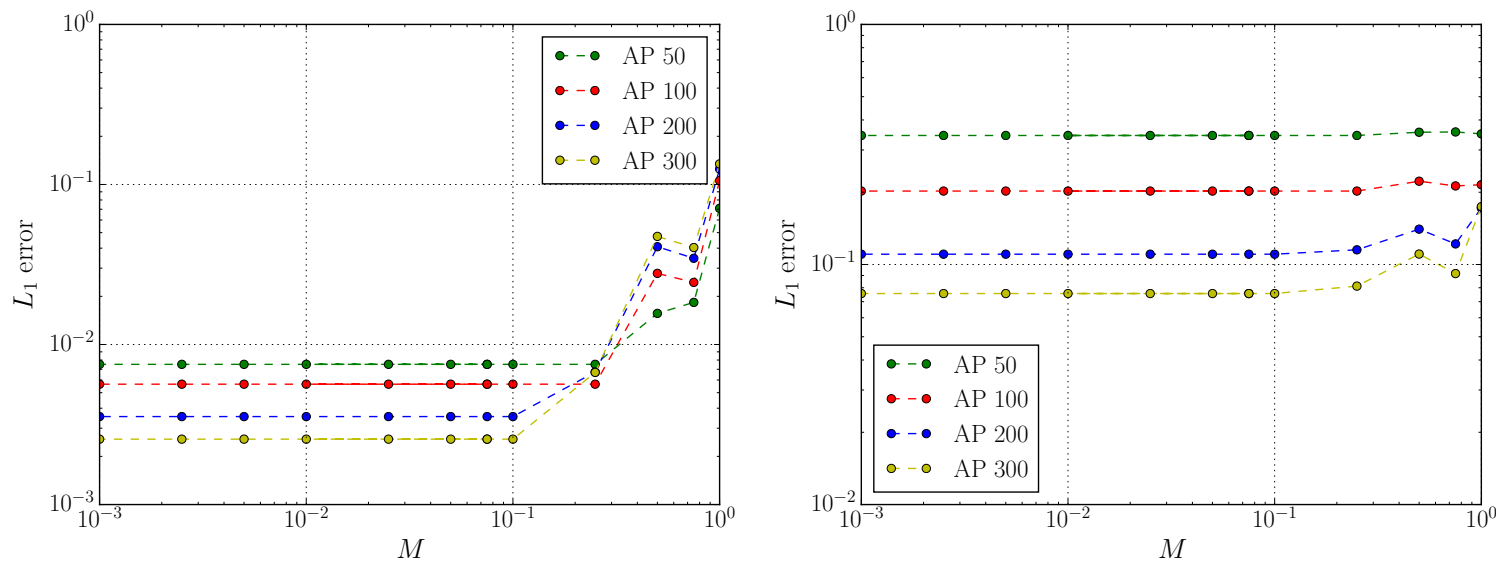

Figure 19: Density error (left) and velocity magnitude error (right) in terms of $M$ for the AP scheme at time $t=1$ for different meshes (Taylor-Green vortex).
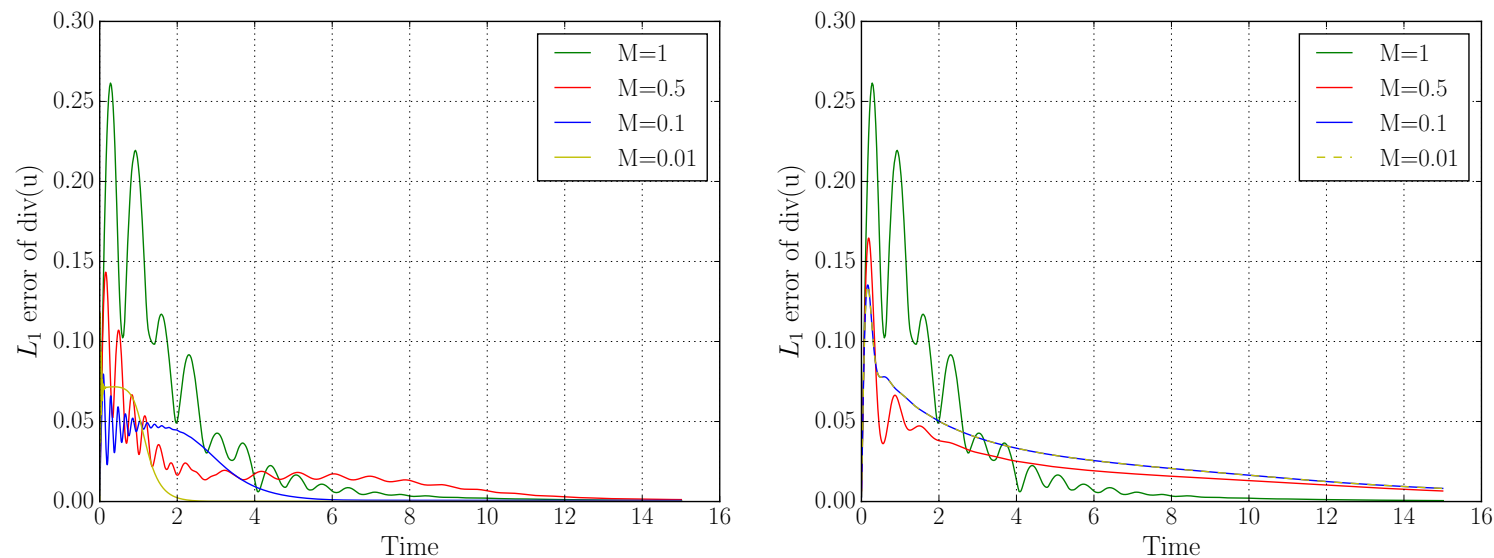

42

Figure 20: Temporal evolution of the relative $L_{1}$ error of the velocity divergence for the relaxation scheme (left) and the AP scheme (right) in the case $50 \times 50$ (Taylor-Green vortex). 

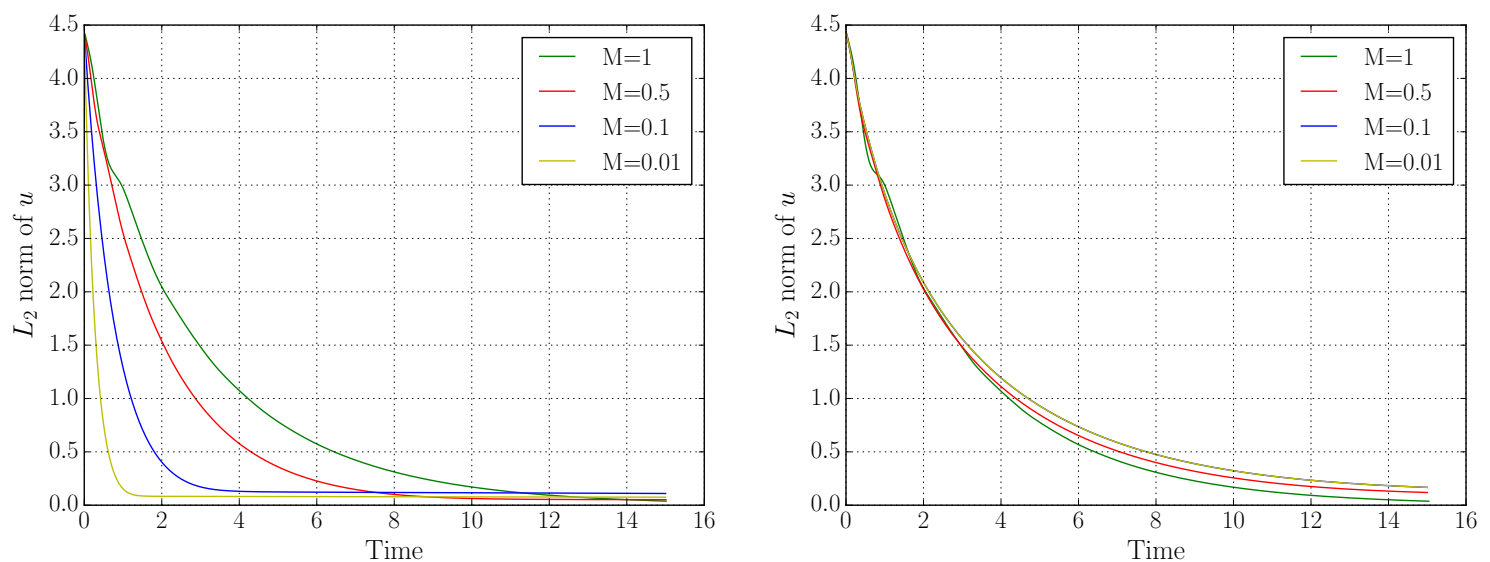

Figure 21: Temporal evolution of the $L_{2}$ norm of the velocity for the relaxation scheme (left) and the AP scheme (right) in the case $50 \times 50$ for different $M$ (Taylor-Green vortex).
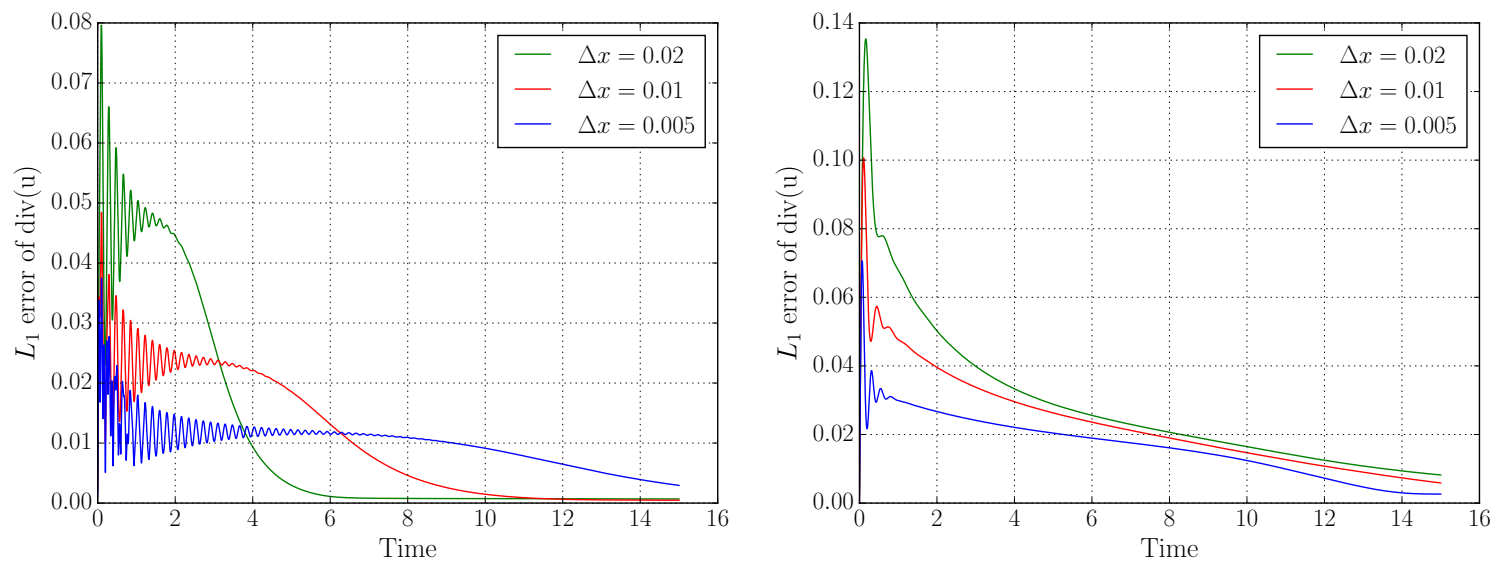

Figure 22: Temporal evolution of the $L_{1}$ error of the velocity divergence for the relaxation scheme (left) and the AP scheme (right) in the case $M=0.1$ for different meshes (Taylor-Green vortex). 

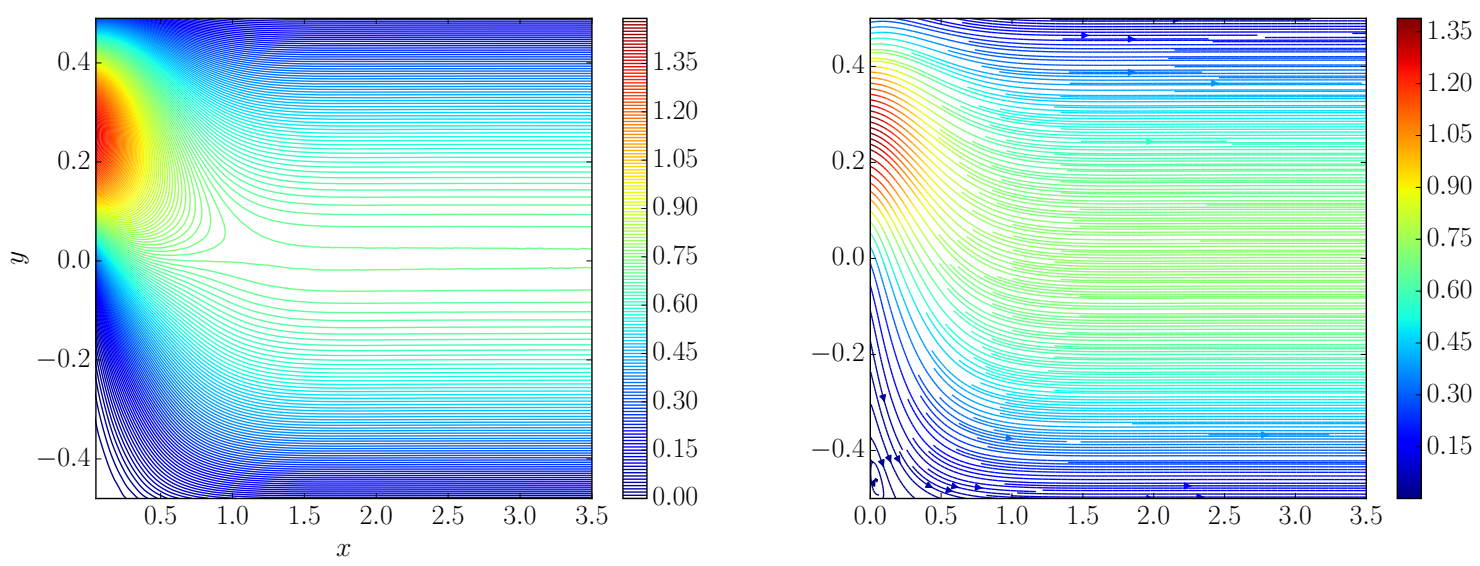

Figure 23: Contour plots of the velocity modulus (left) and velocity stream functions (right) for the Rusanov scheme in the case $\Delta x=1 / 16$ and $\Delta y=$ $1 / 32$ for $M=0.01$ and $R e=100$ at time $t=20$.
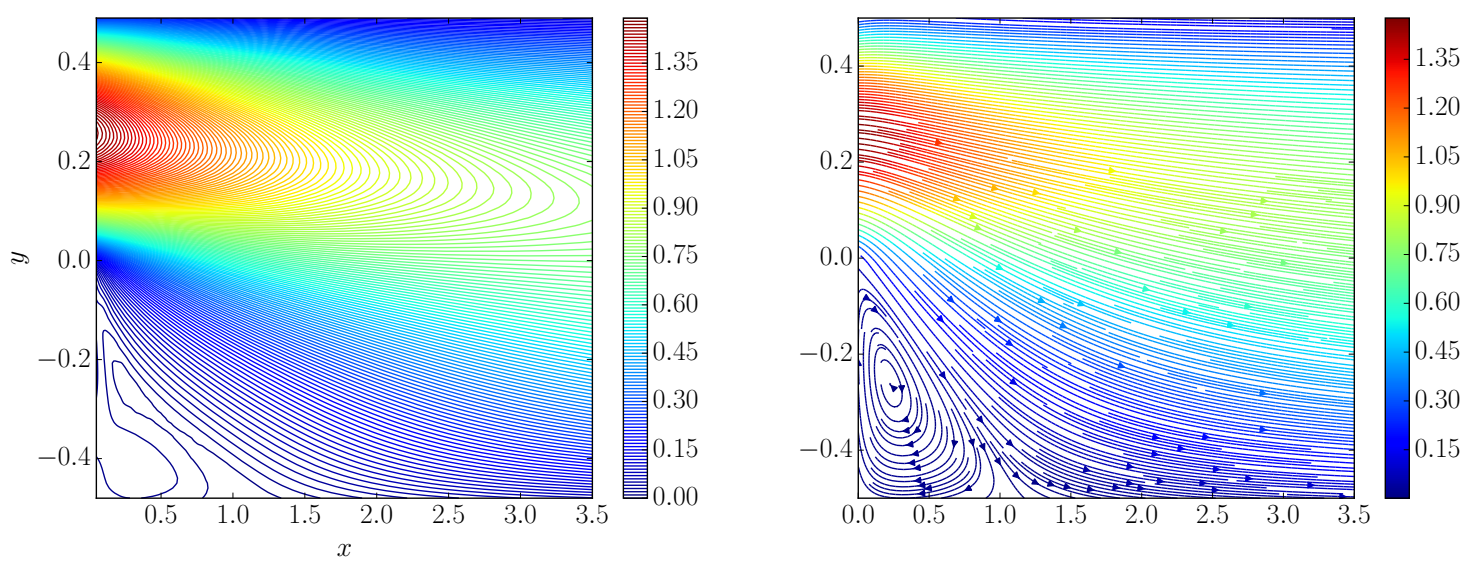

Figure 24: Contour plots of the velocity modulus (left) and velocity stream functions (right) for the relaxation scheme in the case $\Delta x=1 / 16$ and $\Delta y=$ $1 / 32$ for $M=0.01$ and $R e=100$ at time $t=20$.
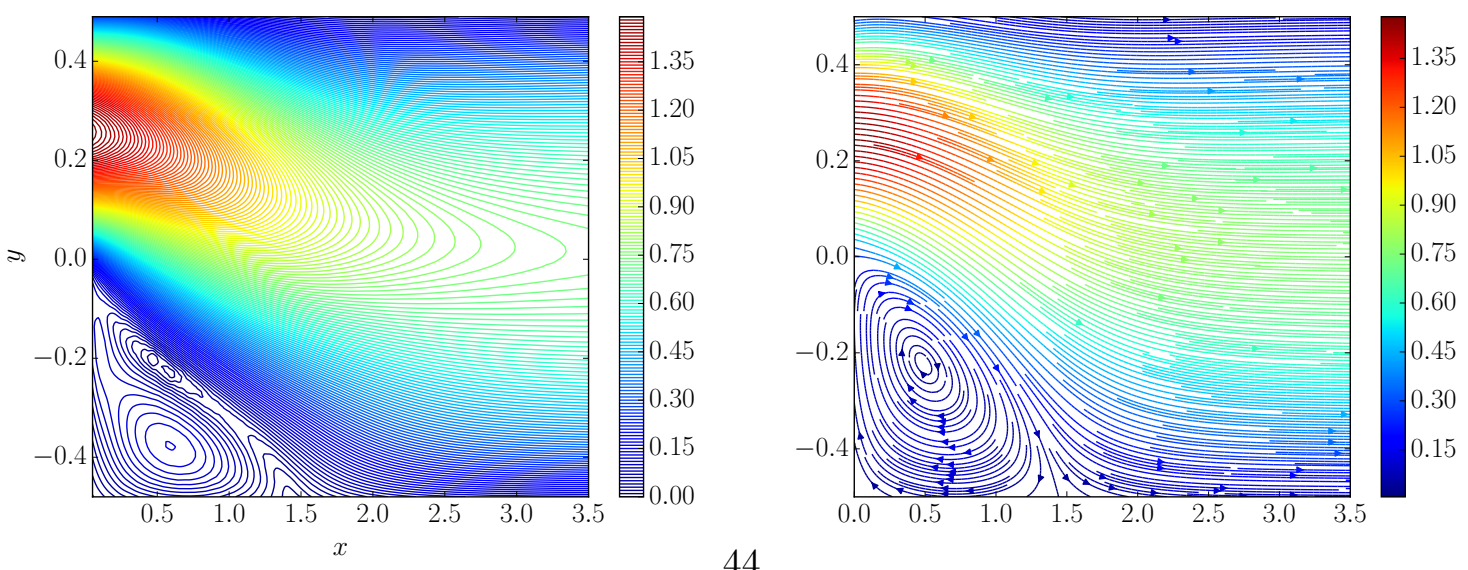

Figure 25: Contour plots of the velocity modulus (left) and velocity stream functions (right) for the AP scheme in the case $\Delta x=1 / 16$ and $\Delta y=1 / 32$ for $M=0.01$ and $R e=100$ at time $t=20$. 

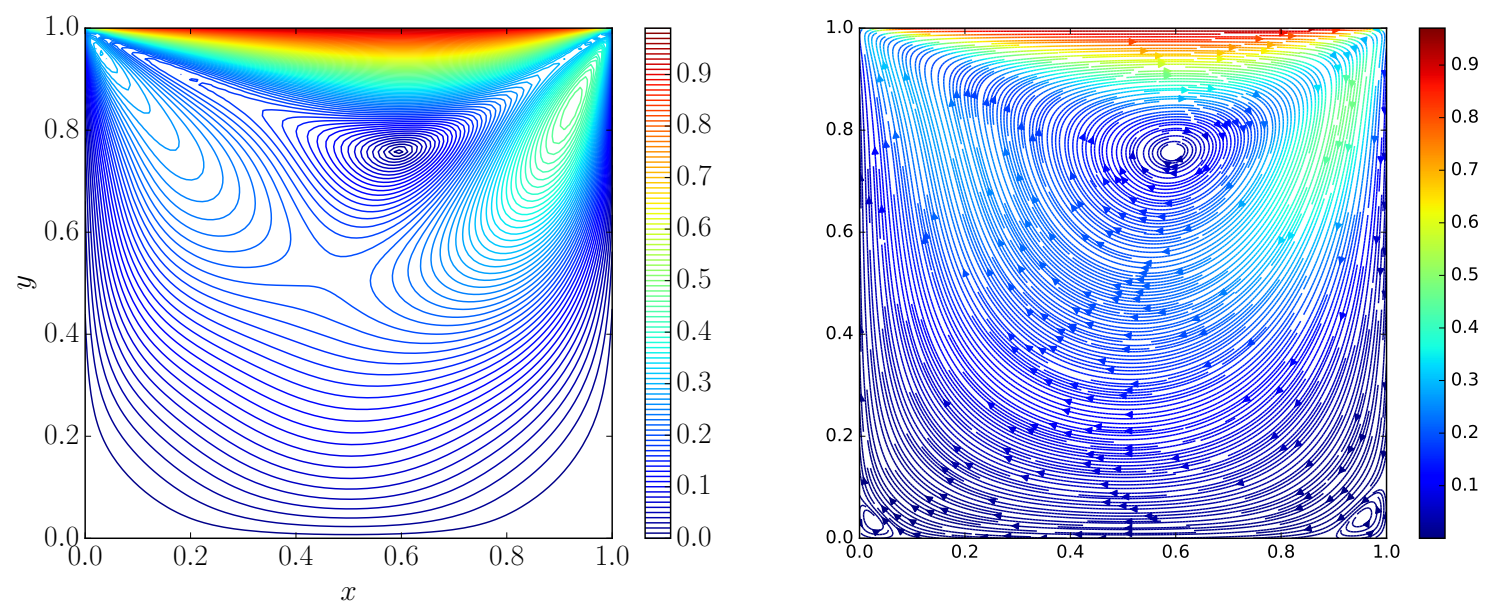

Figure 26: Contour plots of the velocity modulus (left) and velocity stream functions (right) for the Rusanov scheme in the case $\Delta x=\Delta y=1 / 200$ for $M=0.2$ and $R e=400$ at time $t=30$.
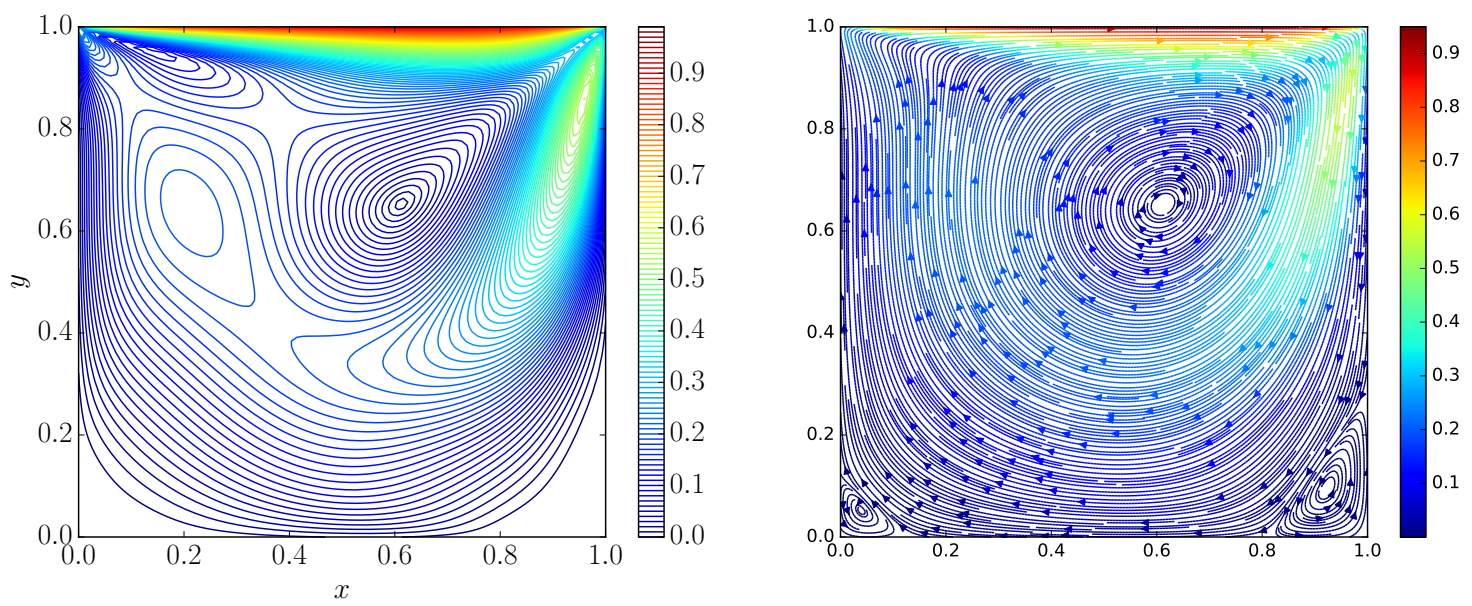

Figure 27: Contour plots of the velocity modulus (left) and velocity stream functions (right) for the relaxation scheme in the case $\Delta x=\Delta y=1 / 200$ for $M=0.2$ and $R e=400$ at time $t=30$.
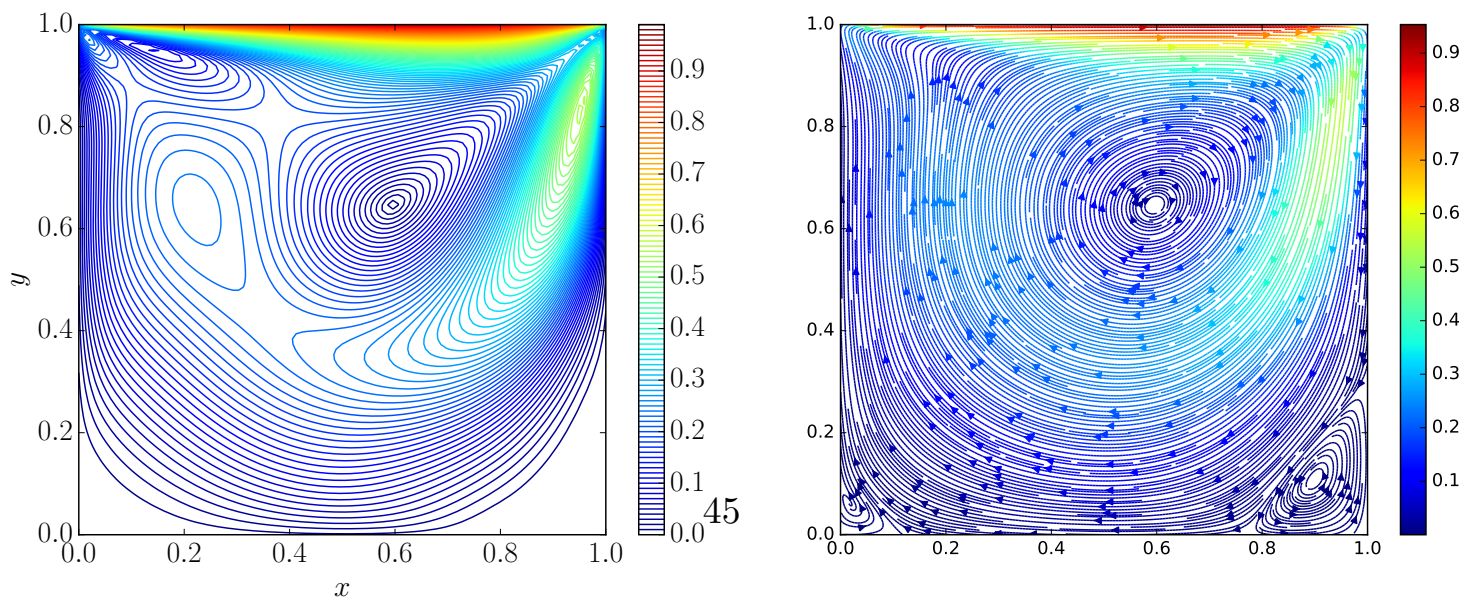

Figure 28: Contour plots of the velocity modulus (left) and velocity stream functions (right) for the AP scheme in the case $\Delta x=\Delta y=1 / 200$ for $M=0.2$ and $R e=400$ at time $t=30$. 

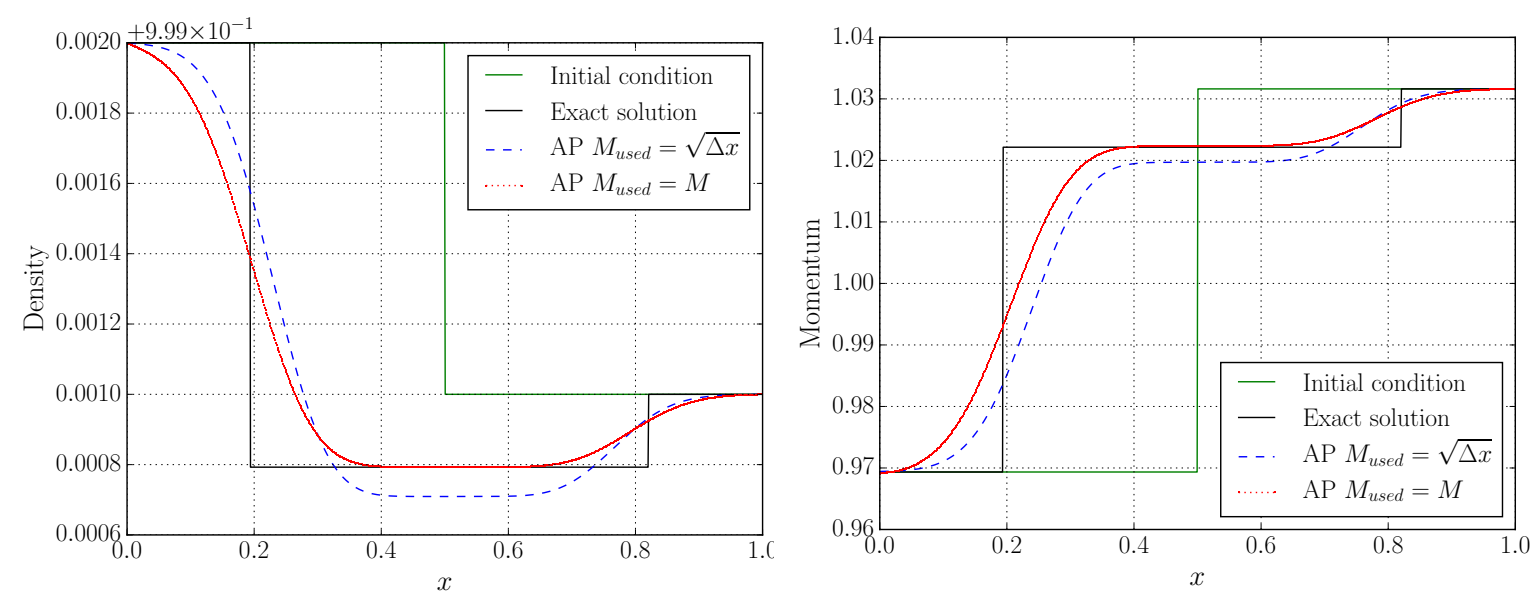

Figure 29: Limit of the approach: representation of the density (left) and momentum (right) at time $t=7 \times 10^{-3}$ in the case $M^{2}=10^{-3}$ for $M_{\text {used }}=$ $\sqrt{\Delta x}$ (blue) and $M_{\text {used }}=M$ (red). 